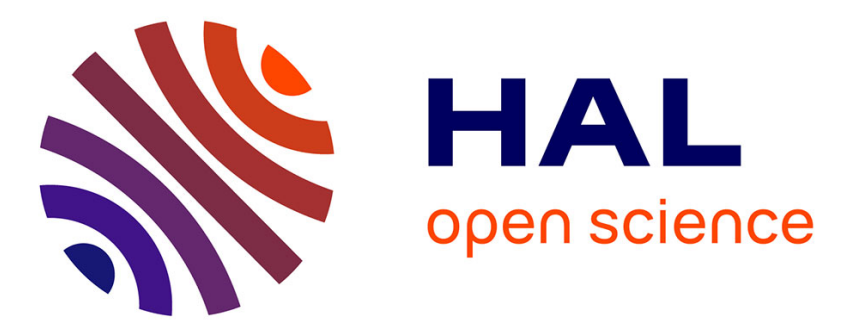

\title{
Fast Fourier transform-based micromechanics of interfacial line defects in crystalline materials
}

\author{
Stéphane Berbenni, Vincent Taupin
}

\section{To cite this version:}

Stéphane Berbenni, Vincent Taupin. Fast Fourier transform-based micromechanics of interfacial line defects in crystalline materials. Journal of Micromechanics and Molecular Physics, 2019, 03 (03n04), pp.1840007. 10.1142/S2424913018400076 . hal-02351498

\section{HAL Id: hal-02351498 \\ https://hal.science/hal-02351498}

Submitted on 14 Nov 2019

HAL is a multi-disciplinary open access archive for the deposit and dissemination of scientific research documents, whether they are published or not. The documents may come from teaching and research institutions in France or abroad, or from public or private research centers.
L'archive ouverte pluridisciplinaire HAL, est destinée au dépôt et à la diffusion de documents scientifiques de niveau recherche, publiés ou non, émanant des établissements d'enseignement et de recherche français ou étrangers, des laboratoires publics ou privés. 


\title{
Fast Fourier Transform-based micromechanics of interfacial line defects in crystalline materials
}

\author{
Stéphane Berbenni *† \\ Université de Lorraine, CNRS, Arts et Métiers ParisTech \\ LEM3, F-57000 Metz, France \\ Vincent Taupin \\ Université de Lorraine, CNRS, Arts et Métiers ParisTech \\ LEM3, F-57000 Metz, France \\ Received date \\ Accepted date
}

\begin{abstract}
Spectral methods using Fast Fourier Transform (FFT) algorithms have recently seen a surge in interest in the mechanics community. The present contribution addresses the critical question of determining local mechanical fields using the FFT method in the presence of interfacial defects. Precisely, the present work introduces a numerical approach based on intrinsic discrete Fourier transforms for the simultaneous treatment of material discontinuities arising from the presence of disclinations, i.e. rotational discontinuities, and inhomogeneities. A centered finite difference scheme for differential rules are first used for numerically solving the Poisson-type equations in the Fourier space to get the incompatible elastic fields due to disclinations and dislocations. Second, centered finite differences on a rotated grid are chosen for the computation of the modified Fourier-Greens operator in the Lippmann-Schwinger-Dyson type equation for heterogeneous media. Elastic fields of disclination dipole distributions interacting with inhomogeneities of varying stiffnesses, grain boundaries seen as DSUM (Disclination Structural Unit Model), grain boundary disconnection defects, and, phase boundary "terraces" in anisotropic bi-materials are numerically computed as applications of the method.
\end{abstract}

Keywords disclinations; dislocations; disconnections; inhomogeneities; anisotropic; grain boundary; numerical method; FFT

\section{Introduction}

Interfaces play a critical role on the mechanical properties of many engineering materials. Continuum micromechanics based modeling of interfacial defects is a fundamental issue to predict the mechanical behavior of nanocrystalline materials and to understand the mechanical interactions between bulk and interfacial defects. The paper deals with a continuous description of elasto-plastic materials with bulk and

*Corresponding author. Email address: stephane.berbenni@univ-lorraine.fr

${ }^{\dagger}$ This paper is dedicated to the memory of Prof. Gérard A. Maugin. 
interfacial defects combining both dislocation and disclination mechanics to model grain boundaries and phase boundaries in anisotropic and heterogeneous materials.

Dislocations and disclinations were mathematically introduced in a linear elastic medium by Volterra as displacement and rotation discontinuities respectively [Volterra, 1907]. If only dislocations are present in the body, the discontinuity of the (elastic) displacement vector (the so-called Burgers vector) derives from the incompatibility of the elastic distortion (i.e. lattice incompatibility), whereas the elastic curvature tensor remains compatible. This incompatibility was described smoothly in the elastic theory of continuum-based dislocation mechanics originated from the fifties [Kröner, 1958; Bilby et al., 1955; Mura, 1963; Willis, 1967; Lardner, 1969; Simmons and Bullough, 1970; Kröner, 1981; Teodosiu, 1982; Mura, 1987]. This continuous rendition of incompatibilities uses the dislocation density tensor introduced by Nye [1953] and Kröner [1955, 1958]. The Green's function method was used to derive the so-called Mura-Willis formula which gives the elastic distortion in an infinite homogeneous anisotropic medium as function of dislocation density tensor, see e.g. [Mura, 1987; Lazar, 2016]. The continuum dislocation theory was also revisited by Acharya [2001] through the so-called Field Dislocation Mechanics (FDM) theory. The finite element formulation of the FDM theory was reported in Roy and Acharya [2005].

In crystalline solids, the presence of disclinations in addition to dislocations leads to (elastic) multi-valued displacement and rotation vectors [Anthony et al., 1968; Anthony, 1970; deWit, 1970, 1973a; Huang and Mura, 1969; Mura, 1972, 1987]. Therefore, in the presence of disclinations, both the elastic strain and the elastic curvature tensors are incompatible. The disclination density tensor was first introduced and discussed in the contributions by Anthony et al. [1968], Schaefer [1968] and deWit [1970]. Later, a mathematical theory for disclination dynamics was provided in Kossecka and DeWit [1977]. A non linear theory of dislocations and disclinations was reported by Zubov [1997]. More recently, the Field Dislocation and Disclination Mechanics (FDDM) theory was introduced as an extension of the FDM theory to non-local fields due to both disclination and dislocation defects that are smooth over an interatomic length scale [Fressengeas et al., 2011; Upadhyay et al., 2013; Fressengeas et al., 2014; Taupin et al., 2017]. The finite element formulation of the FDDM theory was reported in Fressengeas et al. [2011] and Taupin et al. [2013]. One of the key features of the FDM and FDDM theories in contrast with classic micromechanical methods based on eigenstrains is the Stokes-Helmholtz decomposition of the elastic fields into incompatible and compatible parts. The incompatible part is related to the presence of non-zero dislocation and disclination densities within the body, while the compatible part is needed to ensure balance of the stress field and boundary conditions. Such a decomposition allows determining a unique solution for the elastic fields associated with prescribed dislocation and 
disclination densities. Therefore, it provides a rigorous basis for the description of the incompatibility of the elastic strain and the elastic curvature due to the presence of bulk and interfacial defects as dislocations and disclinations in crystalline media.

Disclination kinematics was seen to be useful in the case of grain and subgrain boundaries that can be simulated in terms of array of disclination dipoles, see e.g. Li [1972]; Romanov and Vladimirov [1992]; Hurtado et al. [1995]; Seefeldt et al. [2001]; Romanov and Kolesnikova [2009]; Upadhyay et al. [2011]; Fressengeas et al. [2014]; Dingreville and Berbenni [2016]. Elastic fields and energy of grain boundaries were simulated using the Disclination Structural Unit Model (DSUM) [Gertsman et al., 1989; Nazarov et al., 2000; Nazarov and Romanov, 1989]. Disclinations are also commonly invoked to explain plastic deformation in nanocrystalline materials [Gutkin and Ovid'ko, 2003, 2004] and the formation of twin junctions in the case of secondary (or hierarchical) twinning [Müllner and King, 2016]. Triple junctions in polycrystals can also be described by disclinations [Bollmann, 1991]. It is noteworthy that a few contributions studied the mechanical interactions between disclination dipoles and inhomogeneities [Liu et al., 2006]. Elastic fields of disclination dipoles were used to study the internal stresses and rotations in graphene [Wu and Wei, 2013; Ren and Cao, 2016]. Recently, disclination densities were measured by the EBSD technique (Electron Back-Scattered Diffraction) with different post-treatments [Beausir and Fressengeas, 2013; Leff et al., 2017]. Lastly, a combination of dislocation and disclination line defects were used in the literature to model disconnection defects. Such defects are interfacial line defects with dislocation and step characters first introduced by Hirth and Pond [1996, 2011]. Hirth et al. [2006] showed that disconnections can be formed as new interfacial defects during slip transfer at grain boundaries. Khater et al. [2012], Rajabzadeh [2013] and Rajabzadeh et al. [2014] demonstrated that coupled grain boundary migration and shear is based on a disconnection mechanism. Elastic fields of such disconnections calculated in isotropic elasticity were reported in Akarapu et al. [2008]. Some arrays of disconnections can be also used to model martensitic interfaces (habit planes) or hetero-interfaces forming a terrace structure observed with TEM (transmission electron microscopy) [Pond et al., 2003, 2007; Wang et al., 2011]. Numerical calculations with the finite element method (FEM) were provided by Zhang et al. [2018] for such complex defects in small and finite deformation settings considering generalized disclinations [Acharya and Fressengeas, 2012].

Due to computational efficiency as compared to the usual finite element method (FEM), spectral methods taking advantage of the Fast Fourier Transform algorithm [Frigo and Johnson, 1998] were reported mostly to solve the integral equation for periodic heterogeneous elastic media without or with eigenstrains [Moulinec and Suquet, 1994; Müller, 1996; Moulinec and Suquet, 1998; Dreyer et al., 1999; Vinogradov and Milton, 2008; Willot and Pellegrini, 2008; Brisard and Dormieux, 
2010; Anglin et al., 2014; Willot, 2015]. Indeed, the FFT numerical scheme allows solving the so-called Lippmann-Schwinger-Dyson integral equation of the periodic boundary-value problems, by the means of Green's function of a well-chosen reference medium, using an iterative scheme in the Fourier space, while the resulting elastic fields are obtained in the real space by the inverse Fourier transform. The most well known and pioneering iterative scheme is the "basic scheme" based on a fixed-point algorithm as reported in Moulinec and Suquet [1994, 1998] and by Lebensohn [2001]. The convergence of this numerical scheme is based on fulfillment of stress equilibrium in the Fourier space and depends on the elasticity moduli contrast and on the choice of the homogeneous reference medium. Fast Fourier Transform-based micromechanics was recently developed to numerically solve the static FDM theory [Brenner et al., 2014; Berbenni et al., 2014; Djaka et al., 2017] and the dislocation density transport equation [Djaka et al., 2015]. However, the numerical resolution by FFT of the elasto-static equations of FDDM (Field Dislocation and Disclination Mechanics) within a general heterogeneous and anisotropic linear elastic framework has not been explored yet. The difficulty lies in the numerical resolution of the balance of linear momentum (discarding body force and inertial effects), which requires solving an implicit Lippmann-Schwinger-Dyson integral equation in heterogeneous and anisotropic media with incompatible elastic fields arising from the presence of both dislocation and disclination densities. Such resolution is different from the classic Eshelby transformation problem where dislocations were described as eigendistortions [Eshelby, 1957, 1961; Lazar, 2016, 2017] and disclinations as eigenstrains/eigencurvatures [deWit, 1973a] in homogeneous elastic media only. Here, a numerical spectral approach based on the efficient Fast Fourier Transform (FFT) method is developed to accurately solve both dislocation and disclination mechanical fields in heterogeneous media like bi-materials, inclusion-based composite materials.

The paper is organized as follows. Notations are introduced in section 2. The elastostatic field equations of the continuum mechanics of defects (static FDDM equations) in a small deformation setting are introduced in Section 3 as well as the Lippmann-Schwinger-Dyson integral equation with incompatibilities. In section 4 , the static field equations are solved using the FFT-based algorithm. The incompatible and compatible elastic strains, elastic curvatures as well as the stress fields are obtained from the solutions of Poisson and Lippmann-Schwinger-Dyson equations in the Fourier space. The stress fields in the vicinity of the defects are accurately solved by the proposed discrete Fourier transform method, devoid of spurious numerical oscillations. Section 5 displays the numerical applications on different bulk and interfacial line defects: (i) Disclination dipole distributions interacting with inhomogeneities, (ii) Grain boundaries seen as DSUM (Disclination Structural Unit Model) (iii) Disconnection defect and phase boundary terraces in anisotropic bimaterials. Finally, in section 6 , the conclusions of the paper are outlined. 


\section{Notations}

A bold symbol denotes a tensor or a vector. The symmetric part of second order tensor $\mathbf{A}$ is denoted $\mathbf{A}^{\text {sym }}$. When needed, a symmetric tensor will be denoted using component form $A_{(i j)}$ with $A_{i j}=A_{j i}$. The transpose of a second order tensor $\mathbf{A}$ is denoted by $\mathbf{A}^{t}$ with $A^{t}{ }_{i j}=A_{j i}$ using indicial notation. The tensor $\mathbf{A} \cdot \mathbf{B}$, with rectangular Cartesian components $A_{i k} B_{k j}$, results from the dot product of tensors $\mathbf{A}$ and $\mathbf{B}$, and $\mathbf{A} \otimes \mathbf{B}$ is their tensorial product, with components $A_{i j} B_{k l}$. The vector $\mathbf{A} \cdot \mathbf{V}$, with rectangular Cartesian components $A_{i j} V_{j}$, results from the dot product of tensor $\mathbf{A}$ and vector $\mathbf{V}$. A symbol ":" represents the trace inner product of the two second order tensors $\mathbf{A}: \mathbf{B}=A_{i j} B_{j i}$, in rectangular Cartesian components, or the product of a higher order tensor with a second order tensor, e.g., $\mathbf{A}: \mathbf{B}=A_{i j k l} B_{k l}$. In rectangular Cartesian components, the cross product " $\times$ " of a second-order tensor $\mathbf{A}$ and a vector $\mathbf{V}$, the grad of a vector $\mathbf{V}$, the $\operatorname{div}$, curl of $\mathbf{A}$ are given by:

$$
\begin{aligned}
(\mathbf{A} \times \mathbf{V})_{i j} & =e_{j k l} A_{i k} V_{l} \\
(\operatorname{grad} \mathbf{V})_{i j} & =V_{j, i} \\
(\operatorname{div} \mathbf{A})_{i} & =A_{j i, j} \\
(\operatorname{curl} \mathbf{A})_{i l} & =e_{i m k} A_{k l, m}
\end{aligned}
$$

where $e_{p m k}$ is a component of the third-order alternating Levi-Civita tensor and the spatial derivative with respect to a Cartesian coordinate is indicated by a comma followed by the component index. For convenience, the same convention for notations is used as in deWit [1973a].

\section{Continuum theory of defects}

\subsection{Dislocation and disclination densities}

The theory is developed in the framework of linear kinematics (small strains and rotations). Let us consider the elastic curvature tensor $\boldsymbol{\kappa}^{e}$ and the elastic strain tensor $\varepsilon^{e}$ in a body $V$ containing disclination and dislocation-type defects that may be present in crystalline solids and interfaces. Let us define a closed circuit $C$ delimiting a surface $S$ of unit normal $\mathbf{n}$ so that disclination and dislocation lines cross the surface $S$.

In the case where the resulting Frank and Burgers vectors of all threading disclination/dislocation lines are non-zero, there is incompatibility, in the sense that the rotation and displacement fields become multi-valued. A measure of this incompatibility for simply-connected body is precisely the net Frank vector $\boldsymbol{\omega}$ and the net general Burgers vector $\mathbf{b}$ respectively. Here, a $F S / R H$ convention is used for their definitions as in deWit [1973a]. These two quantities are defined along the Burgers 
Authors' Names

circuit $C$ using Weingarten's theorem, see e.g. deWit [1970, 1973a]:

$$
\begin{aligned}
& \boldsymbol{\omega}=\int_{C} \boldsymbol{\kappa}^{e} \cdot \mathbf{d} \mathbf{l} \\
& \mathbf{b}=\int_{C}\left(\varepsilon^{e}-\boldsymbol{\kappa}^{e} \times \mathbf{x}\right) \cdot \mathbf{d} \mathbf{l}
\end{aligned}
$$

Using the component form of Eq. 1 and notations defined in Section 2 yields:

$$
\begin{aligned}
\omega_{i} & =\int_{C} \kappa_{j i}^{e} d l_{j} \\
b_{i} & =\int_{C}\left(\varepsilon_{k i}^{e}-e_{i q r} \kappa_{k q}^{e} x_{r}\right) d l_{k}
\end{aligned}
$$

From Stokes' theorem, Eq. 1 yields:

$$
\begin{aligned}
& \boldsymbol{\omega}=\int_{S} \boldsymbol{\theta} \cdot \mathbf{n} d S \\
& \mathbf{b}=\int_{S}(\boldsymbol{\alpha}-\theta \times \mathbf{x}) \cdot \mathbf{n} d S
\end{aligned}
$$

where $\mathbf{n}$ is the unit normal to the surface $S$ delimited by the circuit $C$. Using the component form of Eq. 1 yields:

$$
\begin{aligned}
\omega_{i} & =\int_{S} \theta_{j i} n_{j} d S \\
b_{i} & =\int_{S}\left(\alpha_{k i}-e_{i q r} \theta_{k q} x_{r}\right) n_{k} d S
\end{aligned}
$$

Therefore, both $\boldsymbol{\theta}$ and $\boldsymbol{\alpha}$ are defined from Eqs. 2 and 4 as:

$$
\begin{aligned}
& \theta_{m i}=e_{m j k} \kappa_{k i, j}^{e} \\
& \alpha_{m i}=e_{m j k}\left(\varepsilon_{(k i), j}^{e}+e_{i p k} \kappa_{i p}^{e}\right)
\end{aligned}
$$

The continuous defect density that is associated with incompatible elastic curvature is the disclination density tensor $\boldsymbol{\theta}$, see e.g. Anthony et al. [1968]. The second order tensor $\boldsymbol{\alpha}$ is the dislocation density tensor [Nye, 1953]. In a Cartesian reference frame $\left(\mathbf{e}_{\mathbf{1}}, \mathbf{e}_{\mathbf{2}}, \mathbf{e}_{\mathbf{3}}\right)$, the components $j, i$ of $\boldsymbol{\theta}\left(\theta_{j i}\right.$ in component form), resp. $\boldsymbol{\alpha}\left(\alpha_{j i}\right)$ provide the net Frank, resp. Burgers, vector in direction $\mathbf{e}_{\mathbf{i}}$ per unit surface of $S$ with a unit line vector along $\mathbf{e}_{\mathbf{j}}$. The following conservation equations immediately follow from Eq. 5:

$$
\begin{aligned}
& \theta_{j i, j}=0 \\
& \alpha_{j i, j}+e_{i k m} \theta_{k m}=0
\end{aligned}
$$

Physically speaking, the first of these equations means that disclinations cannot end inside the body, while the second one means that dislocations can only terminate on disclinations [deWit, 1973a]. Here, we will assume that the disclination and dislocation density tensors $\boldsymbol{\theta}$ and $\boldsymbol{\alpha}$ are prescribed periodic fields over the unit cell $V$ to make use of Fast Fourier Transform-based micromechanics in periodic heterogeneous media, see Section 4. 


\subsection{Stokes-Helmholtz orthogonal decomposition}

The elastic fields, namely the elastic strain tensor $\varepsilon^{e}$ and the elastic curvature tensor $\boldsymbol{\kappa}^{e}$, can be decomposed according to the Stokes-Helmholtz orthogonal decomposition. Indeed, there exists a unique periodic tensor field $\chi$ (up to a constant second order tensor) and a unique periodic vector field $\mathbf{w}$ (up to a constant vector) such that $\varepsilon^{e}$ can be written as the sum of the symmetric part of the rotational of $\chi$ and the symmetric part of the gradient of $\mathbf{w}$ :

$$
\varepsilon^{e}=\varepsilon^{e, \perp}+\varepsilon^{e, \|}=(\operatorname{curl} \chi)^{\text {sym }}+(\operatorname{grad} \mathbf{w})^{s y m},
$$

with the orthogonality condition $\int_{V} \operatorname{curl} \chi: \operatorname{grad} \mathbf{w} d V=0$ and where $\varepsilon^{e, \perp}=$ $(\operatorname{curl} \chi)^{\text {sym }}$ and $\varepsilon^{e, \|}=(\operatorname{grad} \mathbf{w})^{\text {sym }}$ represent respectively the incompatible and compatible parts of $\varepsilon^{e}$. In turn, the Stokes-Helmholtz orthogonal decomposition of $\boldsymbol{\kappa}^{e}$ yields:

$$
\kappa^{e}=\kappa^{e, \perp}+\kappa^{e, \|}=\operatorname{curl} \psi+\operatorname{grad} \mathbf{z}
$$

with the orthogonality condition $\int_{V} \operatorname{curl} \boldsymbol{\psi}: \operatorname{grad} \mathbf{z} d V=0$ and where $\boldsymbol{\kappa}^{e, \perp}=$ $\operatorname{curl} \boldsymbol{\psi}$ and $\boldsymbol{\kappa}^{e, \|}=\operatorname{grad} \mathrm{z}$ represent respectively the incompatible and compatible parts of $\boldsymbol{\kappa}^{e}$. Since div curl $\chi=0$ and $\operatorname{div} \operatorname{curl} \boldsymbol{\psi}=0$ :

$$
\begin{aligned}
& \operatorname{div} \boldsymbol{\kappa}^{e, \perp}=0 \\
& \operatorname{div} \varepsilon^{e, \perp}=0
\end{aligned}
$$

By applying the Stokes-Helmholtz decompositions (Eqs. 7 and 8), since $\operatorname{curl} \operatorname{grad} \mathbf{z}=0$ and $\operatorname{curl} \operatorname{grad} \mathbf{w}=0$, Eq. 5 yields:

$$
\begin{aligned}
& \theta_{m i}=e_{m j k} \kappa_{k i, j}^{e, \perp} \\
& \alpha_{m i}=e_{m j k}\left(\varepsilon_{(k i), j}^{e, \perp}+e_{i p k} \kappa_{i p}^{e, \perp}\right)
\end{aligned}
$$

and:

$$
\begin{aligned}
& e_{m j k} \kappa_{k i, j}^{e, \|}=0 \\
& e_{m j k}\left(\varepsilon_{(k i), j}^{e, \|}+e_{i p k} \kappa_{i p}^{e, \|}\right)=0
\end{aligned}
$$

Furthermore, $\varepsilon^{e, \|}, \boldsymbol{\kappa}^{e, \|}$ are respectively given by:

$$
\begin{aligned}
\varepsilon_{(i j)}^{e, \|} & =\frac{1}{2}\left(w_{i, j}+w_{j, i}\right) \\
\kappa_{i j}^{e, \|} & =z_{j, i}=\frac{1}{2} e_{j k l} w_{l, k i}
\end{aligned}
$$

\subsection{Solutions for incompatible fields}

Using the identity $\operatorname{curl} \mathbf{c u r l}=\operatorname{grad} \mathbf{d i v}-\triangle$ where $\Delta$ is the Laplacian and using Eq. 9 , the incompatible elastic curvature and elastic strain are solutions of the following 
Poisson equations:

$$
\begin{aligned}
& \kappa_{j i, k k}^{e, \perp}=-e_{j k l} \theta_{l i, k} \\
& \varepsilon_{(l r), k k}^{e, \perp}=-e_{r q i}\left(\alpha_{i l, q}-e_{l j s} e_{i j k} \kappa_{k s, q}^{e, \perp}\right)_{(l r)}
\end{aligned}
$$

where $(l r)$ denotes a symmetry with respect to $l$ and $r$ indices. Let us note that using the identity $e_{l j s} e_{i j k}=\delta_{s k} \delta_{l i}-\delta_{s i} \delta_{l k}$, the expression of $\varepsilon^{e, \perp}$ can be further simplified to:

$$
\begin{aligned}
& \kappa_{j i, k k}^{e, \perp}=-e_{j k l} \theta_{l i, k} \\
& \varepsilon_{(l r), k k}^{e, \perp}=-e_{r q i}\left(\alpha_{i l, q}-\delta_{i l} \kappa_{p p, q}^{e, \perp}+\kappa_{i l, q}^{t e, \perp}\right)_{(l r)}
\end{aligned}
$$

where $\kappa_{i l}^{t e, \perp}=\kappa_{l i}^{e, \perp}$ and $\kappa_{p p}^{e, \perp}$ is the trace of $\boldsymbol{\kappa}^{e, \perp}$.

Eq. 14 will be transformed in the Fourier space in section 4 and numerically solved using discrete Fourier transforms together with the FFT algorithm. The incompatible elastic fields $\varepsilon^{e, \perp}$ and $\boldsymbol{\kappa}^{e, \perp}$ are strictly related to a prescribed distribution of disclination and dislocation densities $\boldsymbol{\theta}$ and $\boldsymbol{\alpha}$ (source terms) within the periodic unit cell $V$. In contrast, the compatible elastic strain $\varepsilon^{e, \|}$ satisfies the balance of linear momentum, which will be solved by introducing the elastic Green's function method and the associated Lippmann-Schwinger-Dyson equation in heterogeneous and anisotropic media with incompatibilities.

\subsection{Lippmann-Schwinger-Dyson equation: solutions for compatible fields in heterogeneous and anisotropic media with incompatibilities}

In the absence of body force and inertia effects, an overall uniform stress $\bar{\sigma}$ is applied as traction boundary conditions. This corresponds to the spatial average of stress $\langle\boldsymbol{\sigma}\rangle$ over the unit cell $V$ using spatial average theorem. For any periodic stress field $\sigma$, the stress equilibrium equation reads:

$$
\operatorname{div} \sigma=0 \text { in } V .
$$

Here, we consider a linear elastic constitutive law (i.e. Hooke's law) of the form:

$$
\sigma=\mathbf{C}: \varepsilon^{e},
$$

where $\mathbf{C}$ is the fourth order tensor of spatially heterogeneous elastic moduli with classic symmetries for Cartesian components: $C_{i j k l}=C_{j i k l}=C_{i j l k}=C_{k l i j}$.

Using Eq. 7 together with Eq. 16, the balance of linear momentum (Eq. 15) can be rewritten in the form of a heterogeneous Navier equation in $V$ including compatible and incompatible fields:

$$
\operatorname{div} \mathbf{C}: \varepsilon^{e, \|}+\mathbf{f}^{\perp}=0
$$


where the fictitious body force density $\mathbf{f}^{\perp}=\operatorname{div} \mathbf{C}: \varepsilon^{e, \perp}$ reflects the incompatibilities arising from the presence of disclinations and dislocations. Assuming a homogeneous reference medium with linear elastic moduli $\mathbf{C}^{0}$, such that $\mathbf{C}(\mathbf{x})=\mathbf{C}^{0}+\delta \mathbf{C}(\mathbf{x})$ ( $\mathrm{x}$ being a position vector in the unit cell), Eq. 17 yields, in component form:

$$
C^{0}{ }_{i j k l} w_{l, k j}+\tau_{i j, j}=0,
$$

where the stress polarization tensor $\tau$ is introduced and defined as follows:

$$
\boldsymbol{\tau}=\mathbf{C}: \varepsilon^{e, \perp}+\delta \mathbf{C}: \operatorname{grad} \mathbf{w}
$$

In Eq. 19, the first term $\boldsymbol{\sigma}^{\perp}=\mathbf{C}: \varepsilon^{e, \perp}$ represents the stress due to incompatible elastic strains obtained from the resolution of the incompatibility problem only, see Eq. 14. Since $\boldsymbol{\tau}$ contains the unknown compatible elastic strain $\varepsilon^{e, \|}$, Eq. 18 is solved starting from an integral equation as described now. Introducing the Green's function technique, Eq. 18 can be solved in the form of an integral LippmannSchwinger-Dyson equation for the unknown compatible elastic strain $\varepsilon^{e, \|}$, with the additional presence of an incompatible term due to prescribed disclination and dislocation densities:

$$
\varepsilon^{e, \|}(\mathbf{x})=\left\langle\varepsilon^{e, \|}\right\rangle-\left(\Gamma^{0} \star \tau\right)(\mathbf{x}),
$$

where $\star$ denotes spatial convolution product and $\boldsymbol{\Gamma}^{0}$ is the well known "modified" Green's tensor in micromechanics and homogenization theories, which is associated with the homogeneous reference elastic medium $\mathbf{C}^{0}$ [Kröner, 1990; Willis, 1981]. $\left\langle\varepsilon^{e, \|}\right\rangle$ represents the spatial average of $\varepsilon^{e, \|}$ over the unit cell $V$. As shown in Djaka et al. $[2017],\left\langle\varepsilon^{e, \|}\right\rangle$ writes $\left\langle\varepsilon^{e, \|}\right\rangle=\mathbf{C}^{0-1}:(\overline{\boldsymbol{\sigma}}-\langle\boldsymbol{\tau}\rangle)$ where $\langle\boldsymbol{\tau}\rangle$ is the averaged stress polarization over the unit cell $V$. Then, the field solution of Eq. 20 is given by series expansion as:

$$
\varepsilon^{e, \|}(\mathbf{x})=\sum_{n=0}^{+\infty}\left[\left(-\Gamma^{0} \star \delta \mathbf{C}\right)(\mathbf{x})\right]^{n}:\left[\left\langle\varepsilon^{e, \|}\right\rangle-\left(\Gamma^{0} \star \boldsymbol{\sigma}^{\perp}\right)(\mathbf{x})\right] .
$$

The integral equation (Eq. 20) and the traction boundary conditions set an elasticity problem for the unknown field $\varepsilon^{e, \|}$. This equation is similar to the classic integral Lippmann-Schwinger-Dyson equation for elastic composites. Here, we will use in the next section a fixed-point algorithm (the so-called "basic scheme") as in the pioneering work by Moulinec and Suquet [1994] and Moulinec and Suquet [1998] to solve Eq. 21 except for the boundary conditions expressed in terms of tractions. Furthermore, the Poisson equations (Eqs. 13 and 14) for solving $\varepsilon^{e, \perp}$ and $\boldsymbol{\kappa}^{e, \perp}$ are first solved in the Fourier space by FFT from prescribed disclination and dislocation density tensors $\boldsymbol{\theta}$ and $\boldsymbol{\alpha}$, respectively. Hence, the numerical spectral method is different from classic eigenstrain-like problems earlier presented by Vinogradov and Milton [2008] or by Anglin et al. [2014]. Here, the numerical FFT algorithm is based on two different procedures which are (i) the calculation of incompatible elastic fields $\kappa^{e, \perp}$ and $\varepsilon^{e, \perp}$ (ii) the iterative resolution of $\varepsilon^{e, \|}$. 


\section{Numerical spectral approach}

\subsection{Fourier transform method}

The Poisson equations (Eqs. 13, 14) and the series expansion (Eq. 21) are solved in the following by using the Fourier Transform method. Indeed, as shown below, the incompatible fields $\boldsymbol{\kappa}^{e, \perp}, \varepsilon^{e, \perp}$ and the compatible one $\varepsilon^{e, \|}$ can be solved in the Fourier space and the obtained elastic fields are finally computed in the real space by using the inverse Fourier Transform.

In the Fourier space, let $\boldsymbol{\xi}$ be the Fourier vector of magnitude $\xi=\sqrt{\boldsymbol{\xi} \cdot \boldsymbol{\xi}}$ and with components $\xi_{i}$ in Cartesian coordinates. The complex imaginary number is denoted by i and defined as i $=\sqrt{-1}$. Let $\widehat{\boldsymbol{\theta}}(\boldsymbol{\xi}), \widehat{\boldsymbol{\alpha}}(\boldsymbol{\xi}), \widehat{\boldsymbol{\kappa}}^{e, \perp}(\boldsymbol{\xi})$ and $\widehat{\boldsymbol{\varepsilon}}^{e, \perp}(\boldsymbol{\xi})$ be the Fourier transforms of $\boldsymbol{\theta}(\mathbf{x}), \boldsymbol{\alpha}(\mathbf{x}), \boldsymbol{\kappa}^{e, \perp}(\mathbf{x})$ and $\boldsymbol{\epsilon}^{e, \perp}(\mathbf{x})$. Then, the Poisson equations are solved using the differentiation theorem in Fourier space.

Using component notations, Eqs. 13 and 14 write in the Fourier space:

$$
\begin{aligned}
& \widehat{\kappa}_{j i}^{e, \perp}(\boldsymbol{\xi})=\mathrm{i} \frac{\xi_{k}}{\xi^{2}} e_{j k l} \widehat{\theta}_{l i}(\boldsymbol{\xi}) \forall \boldsymbol{\xi} \neq \mathbf{0}, \\
& \widehat{\kappa}_{j i}^{e, \perp}(\mathbf{0})=\mathbf{0} \\
& \widehat{\epsilon}_{(l r)}^{e, \perp}(\boldsymbol{\xi})=\mathrm{i} \frac{\xi_{q}}{\xi^{2}} e_{r q i}\left(\widehat{\alpha}_{i l}(\boldsymbol{\xi})-\delta_{i l} \widehat{\kappa}_{p p}^{e, \perp}(\boldsymbol{\xi})+\widehat{\kappa}_{i l}^{t e, \perp}(\boldsymbol{\xi})\right)_{(l r)} \forall \boldsymbol{\xi} \neq \mathbf{0}, \\
& \widehat{\epsilon}_{(l r)}^{e, \perp}(\mathbf{0})=\mathbf{0},
\end{aligned}
$$

Let $\widehat{\boldsymbol{\varepsilon}}^{e, \|}(\boldsymbol{\xi})$ and $\widehat{\boldsymbol{\Gamma}}^{0}(\boldsymbol{\xi})$ be the continuous Fourier transform of $\boldsymbol{\varepsilon}^{e, \|}(\mathbf{x})$ and $\boldsymbol{\Gamma}^{0}(\mathbf{x})$, respectively. The Fourier transform of the integral Lippmann-Schwinger-Dyson type equation (Eq. 20) yields:

$$
\begin{aligned}
& \widehat{\boldsymbol{\varepsilon}}^{e, \|}(\boldsymbol{\xi})=-\widehat{\boldsymbol{\Gamma}}^{0}(\boldsymbol{\xi}): \widehat{\boldsymbol{\tau}}(\boldsymbol{\xi}) \forall \boldsymbol{\xi} \neq \mathbf{0}, \\
& \widehat{\boldsymbol{\varepsilon}}^{e, \|}(\mathbf{0})=\left\langle\varepsilon^{e, \|}\right\rangle .
\end{aligned}
$$

The Fourier Transform of the modified Green operator $\Gamma^{0}$ can be calculated in Fourier space for anisotropic materials as follows:

$$
\widehat{\Gamma_{i j k l}^{0}}(\boldsymbol{\xi})=\frac{1}{4}\left(\widehat{G_{i k}^{0}} \xi_{j} \xi_{l}+\widehat{G_{i l}^{0}} \xi_{j} \xi_{k}+\widehat{G_{j k}^{0}} \xi_{i} \xi_{l}+\widehat{G_{j l}^{0}} \xi_{i} \xi_{k}\right)
$$

where $\widehat{\mathbf{G}^{0}}$ is the Fourier transform of the elastic Green tensor defined as [Mura, 1987]:

$$
\left\{\begin{array}{l}
\widehat{G_{i j}^{0}}(\boldsymbol{\xi})=\frac{N_{i j}(\boldsymbol{\xi})}{D(\boldsymbol{\xi})} \quad \forall \boldsymbol{\xi} \neq 0 \\
\widehat{G_{i j}^{0}}(\mathbf{0})=\mathbf{0}
\end{array}\right.
$$

where $N_{i j}(\boldsymbol{\xi})$ denotes the rectangular components of the cofactor matrix related to the acoustic tensor $K_{i j}=C_{i j k l}^{o} \xi_{k} \xi_{l}$ and $D(\boldsymbol{\xi})$ is the determinant of $K_{i j}$ [Mura, 1987]. Due to the symmetry properties of $C_{i j k l}^{o}, N_{i j}(\boldsymbol{\xi})$ satisfies: $N_{i j}(\boldsymbol{\xi})=N_{j i}(\boldsymbol{\xi})$, 
therefore $\widehat{G_{i j}^{0}}=\widehat{G_{j i}^{0}}$ and $\widehat{\Gamma_{i j k l}^{0}}=\widehat{\Gamma_{j i k l}^{0}}=\widehat{\Gamma_{i j l k}^{0}}=\widehat{\Gamma_{k l i j}^{0}}$. In the particular case of isotropic elasticity, the components of $\widehat{\boldsymbol{\Gamma}}^{0}(\boldsymbol{\xi})$ are given by:

$$
\begin{aligned}
& \widehat{\Gamma_{i j k l}^{0}}(\boldsymbol{\xi})=\frac{1}{4 \mu^{0} \xi^{2}}\left(\delta_{i k} \xi_{j} \xi_{l}+\delta_{i l} \xi_{j} \xi_{k}+\delta_{j k} \xi_{i} \xi_{l}+\delta_{j l} \xi_{i} \xi_{k}\right) \\
& -\frac{\left(\lambda^{0}+\mu^{0}\right)}{\mu^{0}\left(\lambda^{0}+2 \mu^{0}\right)} \frac{\xi_{i} \xi_{j} \xi_{k} \xi_{l}}{\xi^{4}} .
\end{aligned}
$$

where $\mu^{0}$ and $\lambda^{0}$ are elastic shear and Lamé moduli, respectively.

\subsection{Fast Fourier Transform-based algorithm}

The direct and the inverse Fourier transforms are computed by using the Fast Fourier Transform (FFT) algorithm [Frigo and Johnson, 1998]. The unit cell $V$ is assumed to have spatial dimensions $T_{1}, T_{2}$ and $T_{3}$ along the $\mathbf{e}_{\mathbf{1}}, \mathbf{e}_{\mathbf{2}}$ and $\mathbf{e}_{\mathbf{3}}$ directions, respectively, and is discretized by a regular rectangular grid with $N_{1} \times N_{2} \times N_{3}$ voxels with position vector $\boldsymbol{x}=\left(i_{1} \delta_{1}, i_{2} \delta_{2}, i_{3} \delta_{3}\right)$, where $i_{1}=0 \rightarrow N_{1}-1, i_{2}=0 \rightarrow N_{2}-1$, $i_{3}=0 \rightarrow N_{3}-1$ and $\delta_{1}, \delta_{2}, \delta_{3}$ are the voxel sizes in the $\mathbf{e}_{\mathbf{1}}, \mathbf{e}_{\mathbf{2}}, \mathbf{e}_{\mathbf{3}}$ directions, respectively. For three-dimensional (3D) problems, the total number of FFT grid points is $N_{t o t}=N_{1} \times N_{2} \times N_{3}$ and $\delta=\delta_{1}=\delta_{2}=\delta_{3}$. The discrete Fourier transform (DFT) of a given spatial function $f$ is $\widehat{f}=\mathrm{FFT}(f)$. Its inverse Fourier transform is $f=\mathrm{FFT}^{-1}(\widehat{f})$. In the forthcoming applications (see section 5), we will consider two-dimensional (2D) applications with bulk and interfacial line defects where $N_{t o t}=N_{1} \times N_{2}$ and $\delta=\delta_{1}=\delta_{2}$ for illustration of the method.

The numerical algorithm used to solve the balanced elastic fields associated with any disclination and dislocation density distributions within periodic media is constituted of two major procedures: (i) the initialization of the iterative scheme corresponds to the initialization of $\varepsilon^{e, \|}$ for a macroscopic imposed stress $\bar{\sigma}$ after computing $\widehat{\boldsymbol{\kappa}}^{e, \perp}(\boldsymbol{\xi})$ and $\widehat{\boldsymbol{\varepsilon}}^{e, \perp}(\boldsymbol{\xi})$ using Eq. 22, and, (ii) the global iterative procedure based on the basic scheme (fixed-point algorithm) to solve Eq. 21, where $\varepsilon^{e, \|}$ is calculated after convergence is reached.

Hence, the algorithm works as follows: Once, the disclination density $\boldsymbol{\theta}(\mathbf{x})$ and the dislocation density $\boldsymbol{\alpha}(\mathbf{x})$ are prescribed in the real space, the initialization procedure begins with the computation of $\widehat{\boldsymbol{\theta}}(\boldsymbol{\xi})$ and $\widehat{\boldsymbol{\alpha}}(\boldsymbol{\xi})$ by using direct FFT (step 1). Then, Eq. 22 is used to first obtain $\widehat{\boldsymbol{\kappa}}^{e, \perp}(\boldsymbol{\xi})$ and then $\widehat{\boldsymbol{\varepsilon}}^{e, \perp}(\boldsymbol{\xi})$ in the Fourier space (step 2), and, $\boldsymbol{\varepsilon}^{e, \perp}(\mathbf{x})$ in real space using inverse FFT (step 3). The initial compatible elastic strain $\varepsilon_{0}^{e, \|}$ is taken as the homogeneous elastic solution $\mathbf{C}^{0^{-1}}: \overline{\boldsymbol{\sigma}}$ (step 4). The initialization procedure is concluded by the computation of the initial stress field $\boldsymbol{\sigma}_{0}(\mathbf{x})$ set to $\mathbf{C}(\mathbf{x}):\left(\varepsilon_{0}^{e, \|}+\varepsilon^{e, \perp}(\mathbf{x})\right)$ (step 5).

In the global iterative loop at iteration $(\mathrm{n}+1)$, the stress field known from iteration (n) denoted $\boldsymbol{\sigma}_{n}(\mathbf{x})$, is transformed in the Fourier space by direct FFT to obtain $\widehat{\boldsymbol{\sigma}}_{n}(\boldsymbol{\xi})$ (step 6), and is used to test the convergence criterion based on stress equilibrium in the Fourier space (step 7 ). If the convergence is reached then the iterative 
procedure is stopped, otherwise the polarization stress tensor $\widehat{\boldsymbol{\tau}_{n}}(\boldsymbol{\xi})$ is computed in the Fourier space by calculating the FFT of $\mathbf{C}^{0}: \varepsilon_{n}^{e, \|}$, and subtracting it from $\widehat{\boldsymbol{\sigma}}_{n}(\boldsymbol{\xi})$ (step 8). The compatible elastic strain is computed in the Fourier space at step 9 using Eq. 23 to obtain $\widehat{\boldsymbol{\varepsilon}}_{n+1}^{e, \|}(\boldsymbol{\xi})(\forall \boldsymbol{\xi} \neq \mathbf{0})$ and $\widehat{\boldsymbol{\varepsilon}}_{n+1}^{e, \|}(\mathbf{0})$ is taken as $\left\langle\varepsilon^{e, \|}\right\rangle$ from iteration (n) considering traction boundary conditions as in Djaka et al. [2017]. Then, the compatible elastic strain is given in the real space from inverse FFT (step 10). The latter is used to update the stress field in the real space (step 11). The iterative loop starts again with the updated compatible elastic strain and stress fields until convergence is reached, see Eq. 27.

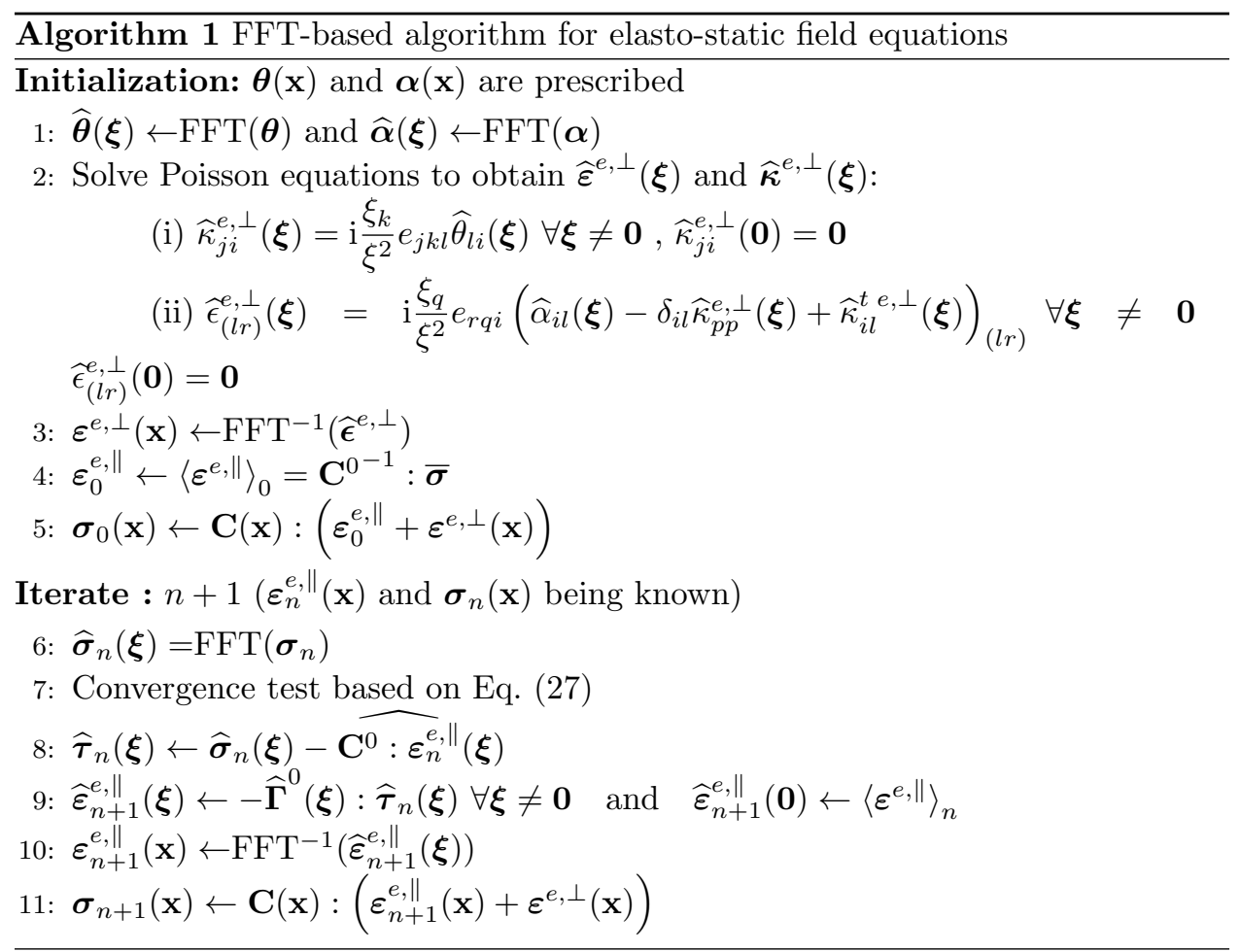

The stress equilibrium convergence criterion used at step 7 reads:

$$
e_{n}=\frac{\left\|\operatorname{div}\left(\boldsymbol{\sigma}_{n}\right)\right\|_{2}}{\left|\left\langle\boldsymbol{\sigma}_{n}\right\rangle\right|}=\frac{\left\|\boldsymbol{\xi} . \widehat{\boldsymbol{\sigma}}_{n}(\boldsymbol{\xi})\right\|_{2}}{\left|\widehat{\boldsymbol{\sigma}}_{n}(\mathbf{0})\right|} \leq \epsilon,
$$

where $\|\cdot\|_{2}$ denotes the $L^{2}$ norm, $|$.$| is the Euclidian norm of a second order tensor$ and $e_{n}$ is the error at iteration $n$. The convergence is reached when the error is smaller than a given precision $\epsilon$ (typically $10^{-5}-10^{-6}$ in our simulations). Then, the elastic strain (incompatible and compatible parts) and stress field are obtained in the real space. 
An important issue inherent to Fourier-based numerical methods is to predict accurate local stress fields near materials discontinuities like defects and inhomogeneities, in particular to avoid spurious oscillations that occur with the use of the classic FFT approximation initially proposed by Moulinec and Suquet [1994, 1998]. To achieve this goal, methods based on finite difference schemes for the computation of partial spatial derivatives of first and second orders were proposed together with the discrete Fourier transform (DFT). A first one based on centered finite difference formulae was used to compute partial derivatives in the Fourier space to solve the elasto-static field equations of linear heterogeneous solids [Müller, 1996; Dreyer et al., 1999]. Later, this method was successfully used to solve the elasto-static fields of FDM in a linear homogeneous isotropic elastic medium with incompatibilities [Berbenni et al., 2014], in a couple stress medium with generalized disclinations [Berbenni et al., 2016], and, in the elasto-viscoplastic FFT algorithm for polycrystals with non local plasticity [Lebensohn and Needleman, 2016]. It was observed that such "intrinsic" DFT technique allows avoiding spurious oscillations that occur with the use of the classic FFT approximation, especially when narrow defect cores, possibly assigned on a single pixel, are considered [Berbenni et al., 2014]. Other "intrinsic" discrete Fourier transforms (DFT) schemes based on forward and backward finite differences were first introduced by Willot and Pellegrini [2008] for elastic-perfectly plastic porous media. A recent scheme for the computation of partial derivatives, based on centered finite differences on a rotated grid, was proposed by Willot [2015] to compute the discrete Green operator present in the LippmannSchwinger-Dyson equation. Such a scheme, referred to as "rotated scheme", was shown to be efficient to give accurate local fields, devoid of spurious oscillations when computing the local stress/strain fields in very crude situations, such as cubic inclusions or voids embedded in a matrix phase. In the case of dislocations only, an efficient and accurate method named $P_{C}-L S_{R}$ was developed to combine a DFT approximation with a centered finite difference scheme for second order derivatives in the Poisson equations $\left(P_{C}\right)$ and a DFT approximation with a "rotated scheme" for solving the Lippmann-Schwinger-Dyson equation $\left(L S_{R}\right)$. The details of this approximation were given in [Djaka et al., 2017]. Here, the $P_{C}-L S_{R}$ approximation is reutilized for the numerical applications presented in section 5 .

\section{Numerical applications}

\subsection{Disclination dipole distributions interacting with inhomogeneities}

A first application of the FFT-based method using the $P_{C}-L S_{R}$ procedure for disclination and dislocation mechanics is the case of a centered wedge disclination dipole with arm length of the dipole $a$ (total length: $2 a$ ) interacting with a circular inhomogeneity $I$ with radius $R=80 \delta$, see Fig. 1 . Assuming heterogeneous and isotropic elasticity, the shear modulus of the inhomogeneity is denoted $\mu_{I}$ whereas 
the shear modulus of the embedding matrix is denoted $\mu_{M}$. The linear elastic behavior of the matrix phase $M$ is isotropic with Young's modulus $E_{M}=62780 \mathrm{MPa}$ and Poisson ratio $\nu_{M}=0.3647$. The elastic Poisson ratio of the inhomogeneity $I$ is the same as the one of the matrix, i.e. $\nu_{I}=\nu_{M}=0.3647$. The mechanical contrast $k$ between the inhomogeneity and the matrix is $k=E_{I} / E_{M}=\mu_{I} / \mu_{M}$ with $\mu_{I}$ and $\mu_{M}$ denoting the shear moduli of inhomogeneity and matrix phases, respectively. For illustrations, studied mechanical contrasts will be $k=1, k=0.1$ and $k=10$. With such elastic constants, the value for the Young's modulus of the reference medium for the iterative basic scheme is given by $E_{0}=\beta\left(E_{M}+E_{I}\right)$ with $\beta=0.505$ as in Djaka et al. [2017]. For a pure positive straight wedge disclination (using the FS/RH convention), both the Frank and line vectors are along the $\mathbf{e}_{\mathbf{3}}$ axis, so the only non zero prescribed disclination density tensor component is $\theta_{33}$ defined as (see Eq. 3):

$$
\omega=\omega_{3}=\int_{S} \theta_{33} d S
$$

where $\omega$ is the Frank's vector magnitude, i.e. the strength of the disclination. Therefore, for the centered wedge disclination dipole with arm length $a$ as represented in Fig. 1, the disclination densities are prescribed on one pixel at both poles as follows: $\theta_{33}=+\omega / \delta^{2}$ at location $\left(x_{1}=0, x_{2}=+a\right)$ and $\theta_{33}=-\omega / \delta^{2}$ at location $\left(x_{1}=0, x_{2}=-a\right)$ with $\omega=5 / 6 \mathrm{rad}$ and $a=2 \delta$. Furthermore, for these simulations, a large unit cell has been discretized using a $2 \mathrm{D}$ computation grid constituted of $1024 \times 1024$ pixels, with a pixel size $\delta=1 \mathrm{~nm}$. The numerical convergence of the FFT-based algorithm is achieved when the error $\epsilon$ in Eq. 27 is smaller than $10^{-6}$. This is the case after $1,39,55$ iterations for $k=1, k=0.1$ and $k=10$, respectively. Here, internal stresses due to the defects are computed. In order to avoid a null denominator in the stress-based convergence criterion (Eq. 27), a slight non zero macroscopic stress $\bar{\sigma}_{11}=1 \mathrm{MPa}$ is imposed as traction boundary conditions without any consequence on the reported values for stress profiles due to the defect. For $k=1$, the simulated normal and shear stress components $\sigma_{11}$ and $\sigma_{12}$ normalized by $D \omega$ with $D=\frac{\mu_{M}}{2 \pi\left(1-\nu_{M}\right)}$ are reported in Fig. 2. These $2 \mathrm{D}$ stress profiles are consistent with analytical solutions [deWit, 1973b; Romanov and Vladimirov, 1992] and with previous FFT-based simulations using homogeneous isotropic elasticity [Berbenni et al., 2014]. The stresses due to mechanical interactions between the circular inhomogeneity and the wedge disclination dipole is more complex for a mechanical contrast $k$ different from 1 . In these cases, analytical formulae in isotropic elasticity classically used for wedge disclination dipoles do not hold and this is why the present FFT-based numerical procedure was developed. For example, Fig. 3 reports the two-dimensional profiles in the $\left(x_{1}, x_{2}\right)$ plane simulated by the FFT-based method for the normal stress component $\sigma_{11}$ (top) and the shear stress component $\sigma_{12}$ (bottom) both normalized by $D \omega$ in the case of $k=0.1$, i.e. an inhomogeneity more compliant than the matrix. In contrast, Fig. 4 reports the 2D profiles for 
the normal stress component $\sigma_{11}$ (top) and the shear stress component $\sigma_{12}$ both normalized by $D \omega$ in the case of $k=10$ (an inhomogeneity stiffer than the matrix).

These results show that the numerical method using periodic boundary conditions inherent to the FFT-based method and disclination dipole gives profiles and internal stress magnitudes as those obtained by the analytical method considering linear elasticity and equivalent edge dislocation with an infinite medium embedding the inhomogeneity, see e.g. Hirth and Lothe [1982]; Dundurs and Sendeckyj [1965]. Generally, the equivalent Burgers vector magnitude $b$ for this edge dislocation is given by the equivalence $a=b /(4 \tan (\omega / 2))$ [Li, 1972; Romanov and Vladimirov, 1992] and similar 2D profiles can be obtained using classic dislocation formulas. However, here the interest was to offer the possibility to provide a numerical formulation using disclination density sources instead of dislocation sources.

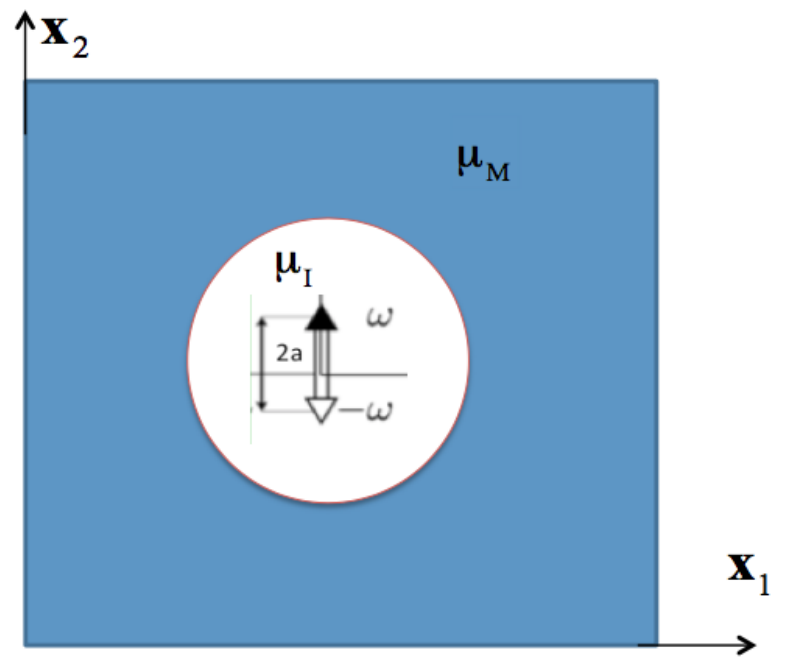

Fig. 1. Configuration representing a wedge disclination dipole interacting with a circular inhomogeneity. 


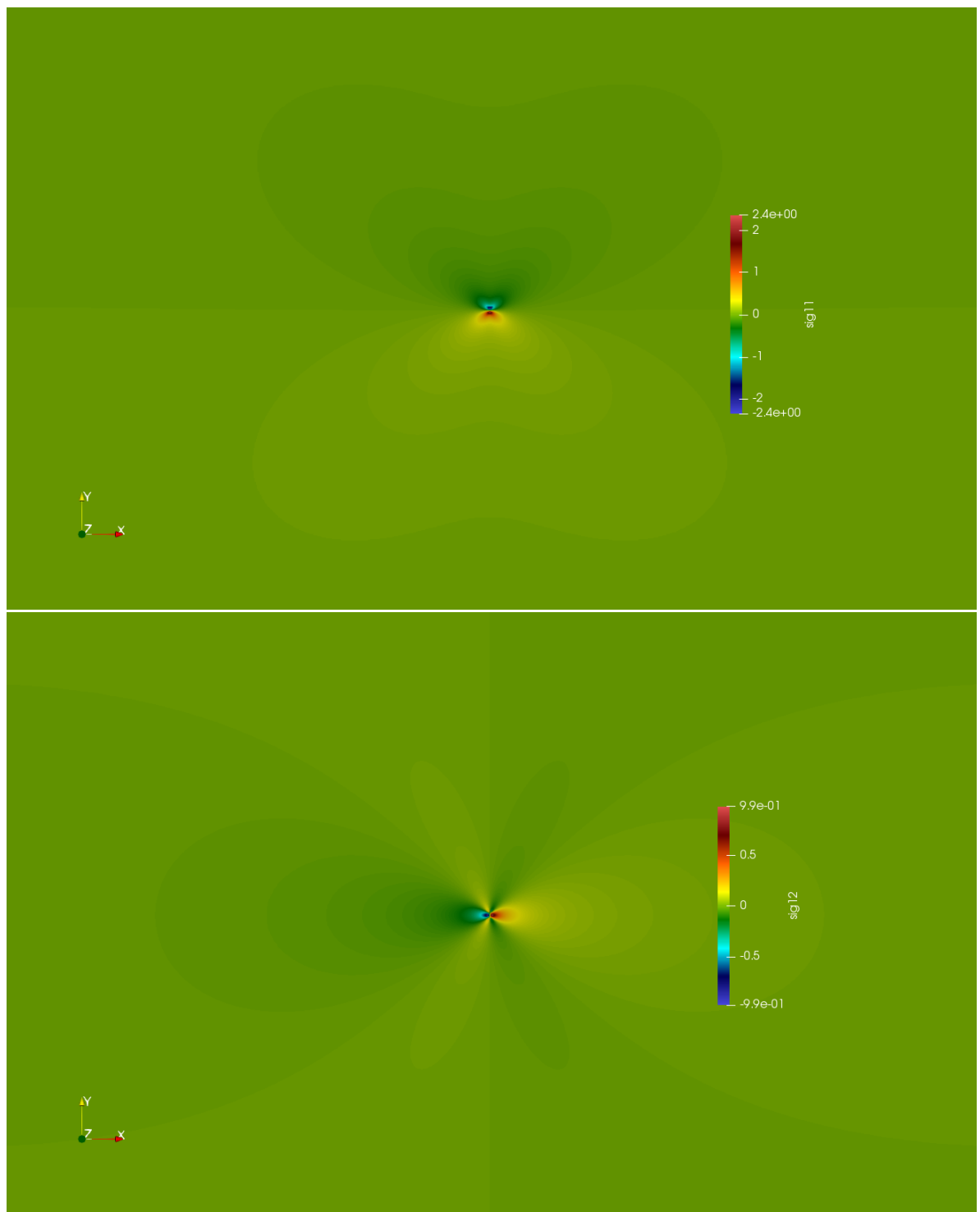

Fig. 2. Normal and shear stress components $\sigma_{11}$ (top) and $\sigma_{12}$ (bottom) normalized by $D \omega$ (with $\left.D=\frac{\mu_{M}}{2 \pi\left(1-\nu_{M}\right)}\right)$ are simulated by FFT-based method with a resolution of $1024 \times 1024$ pixels and disclination density $\theta_{33}$ specified in the text. The elastic moduli contrast $k$ between the inhomogeneity and the matrix is $k=1$.

\subsection{Grain boundaries seen as DSUM (Disclination Structural Unit Model)}

Grain boundaries are constructed using the disclination structural unit model (DSUM) originally proposed by Li [1972] and later improved by Gertsman et al. 


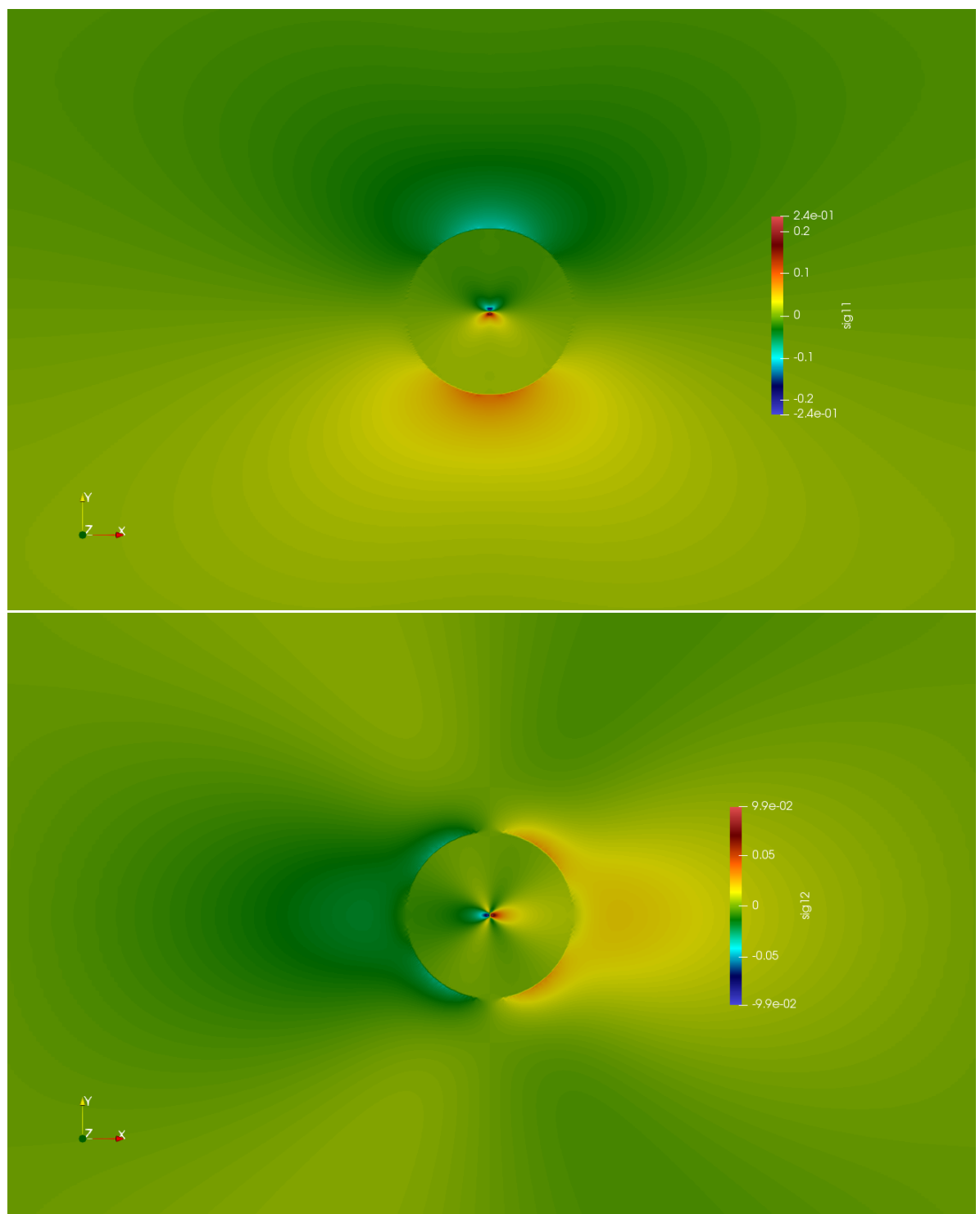

Fig. 3. Normal and shear internal stress components $\sigma_{11}$ (top) and $\sigma_{12}$ (bottom) normalized by $D \omega\left(\right.$ with $\left.D=\frac{\mu_{M}}{2 \pi\left(1-\nu_{M}\right)}\right)$ are simulated by FFT-based method with a resolution of $1024 \times 1024$ pixels and disclination density $\theta_{33}$ specified in the text. The elastic moduli contrast $k$ between the inhomogeneity and the matrix is $k=0.1$ for which 39 iterations were required to reach an error $\epsilon$ less than $10^{-6}$.

[1989]. The DSUM constructs a grain boundary with a misorientation angle $\theta$ by decomposing it into a contiguous and alternating sequence of special (favored) $M$ "majority" and $N$ "minority" structural units with an associated misorientation $\theta_{M}$ and $\theta_{N}$ respectively such that: $\theta_{M}<\theta<\theta_{N}$. In the DSUM, the grain boundaries 


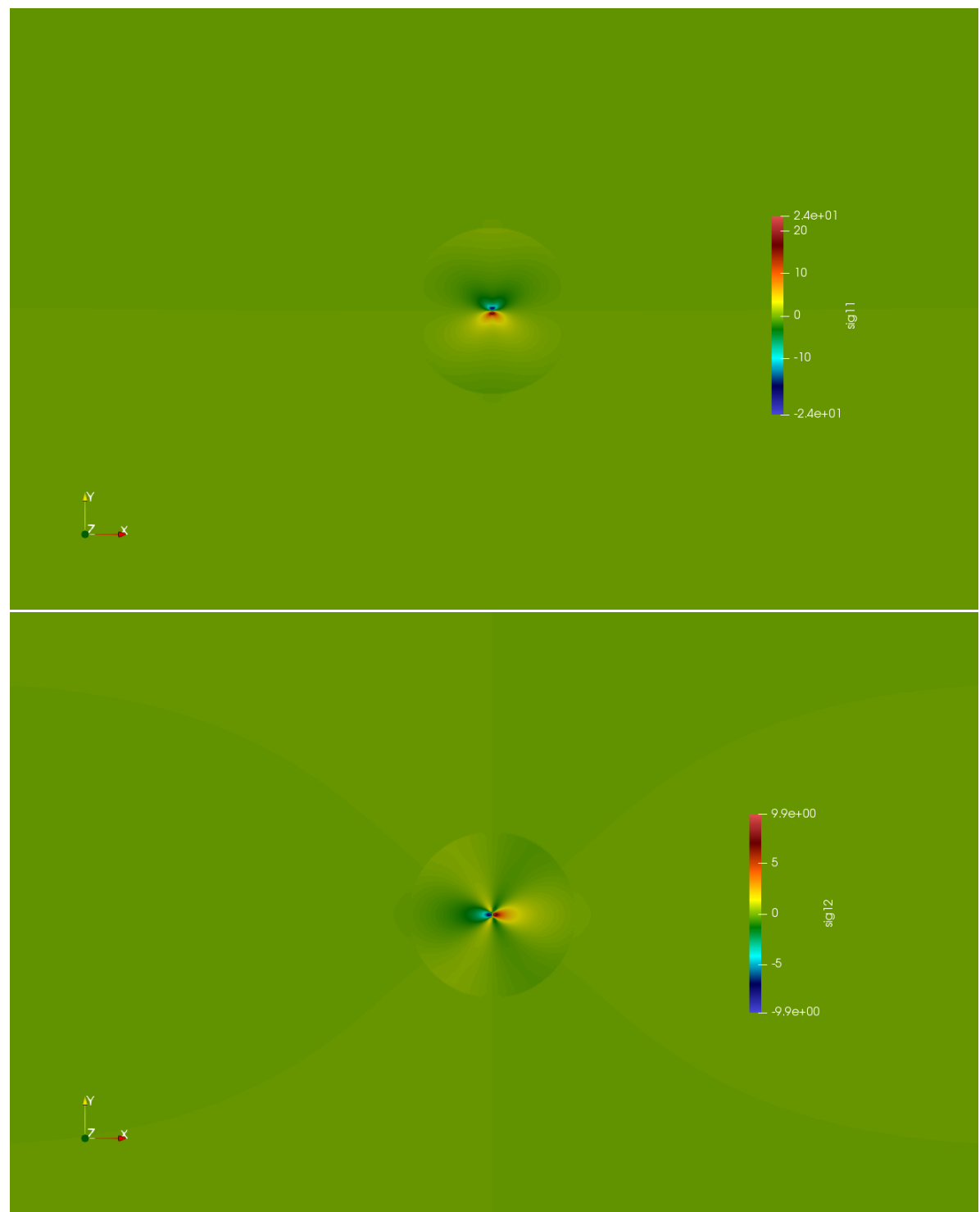

Fig. 4. Normal and shear internal stress components $\sigma_{11}$ (top) and $\sigma_{12}$ (bottom) normalized by $D \omega\left(\right.$ with $\left.D=\frac{\mu_{M}}{2 \pi\left(1-\nu_{M}\right)}\right)$ are simulated by FFT-based method with a resolution of $1024 \times 1024$ pixels and disclination density $\theta_{33}$ specified in the text. The elastic moduli contrast $k$ between the inhomogeneity and the matrix is $k=10$ for which 55 iterations were required to reach an error $\epsilon$ less than $10^{-6}$.

(GB) are represented in the form of a wall of wedge disclination dipoles associated with the minority structural units.

As an example, let us consider the case of symmetric tilt GB (STGB) 
$\Sigma 41(540)[001]$ in aluminium $(\mathrm{Al})$ with misorientation angle around the [001] tilt axis $\theta=12.68^{\circ}$ where $0^{\circ} \leq \theta<36.87^{\circ}$. Therefore, the structural unit model (SUM) [Sutton and Vitek, 1983] for this symmetric tilt GB is composed of the sequence $|A A A B . A A A B|$ where $A$ and $B$ are the structural units characterized by $\theta_{M}=\theta_{A}=0^{\circ}$ (perfect lattice) and $\theta_{N}=\theta_{B}=36.87^{\circ}$ (corresponding to a STGB $\Sigma 5(210)[001])$. The lattice parameter for $\mathrm{Al}$ is $a=0.40495 \mathrm{~nm}$. Here, for the DSUM, we consider $M=3, N=1$. Then, the period $H$ of the GB is given by: $H=M \times\left(2 L_{A}\right)+N \times\left(2 L_{B}\right)$ where $L_{A}$ and $L_{B}$ are defined by the geometric relationship [Nazarov and Romanov, 1989]:

$$
\begin{aligned}
L_{A} & =0.5 D_{A} \cos \left(\frac{\theta-\theta_{A}}{2}\right) \\
L_{B} & =0.5 D_{B} \cos \left(\frac{\theta_{B}-\theta}{2}\right)
\end{aligned}
$$

with $D_{A}=a \sqrt{2} / 2$ and $D_{B}=a \sqrt{5} / 2$. Then, the strength of the wedge disclination dipoles is given by $\omega=\theta_{B}-\theta_{A}$, the arm of the dipoles is fixed and set to $L_{B}$. For numerical calculations, $\mathrm{Al}$ is supposed elastically isotropic, hence $k=1$, which means only one iteration is required for stress equilibrium in the FFT-based algorithm. The elastic constants for $\mathrm{Al}$ were supposed to be isotropic and homogeneous: $\mu=26 G P a$ and $\nu=0.35$. To form the STGB, alternating sequences of disclination density tensor component $\theta_{33}= \pm \omega / \delta^{2}$ with period $H$ at distances $\pm L_{B}$ from the center of each dipole along $\mathbf{e}_{2}$ are prescribed. The computational grid is composed of $1024 \times 1024$ pixels with $\delta=H / 64$. In Fig. 5 (top,middle) normal and shear stresses are normalized by $D \omega=\frac{\mu \omega}{2 \pi(1-\nu)}$. It is seen that the stress profiles are similar to the ones given by analytical solutions in isotropic elasticity [Hurtado et al., 1995; Upadhyay et al., 2011; Dingreville and Berbenni, 2016]. In Fig. 5 (bottom), the axial (elastic) strain $\varepsilon_{22}^{e}$ directed along $\mathbf{e}_{2}$ is reported. It is seen that our predictions of internal strain level both regarding the shape of the strain profile and the magnitudes along the GB (from $-0.13 \%$ to $0.13 \%$ ) are consistent with experimental High Resolution TEM (transmission electron microscopy) results found by Rajabzadeh [2013] (see their figure 3.1b on page 50) using the Geometric Phase Analysis (GPA) method for which such axial strain values for same GB varies from $-0.17 \%$ to $0.12 \%$. 


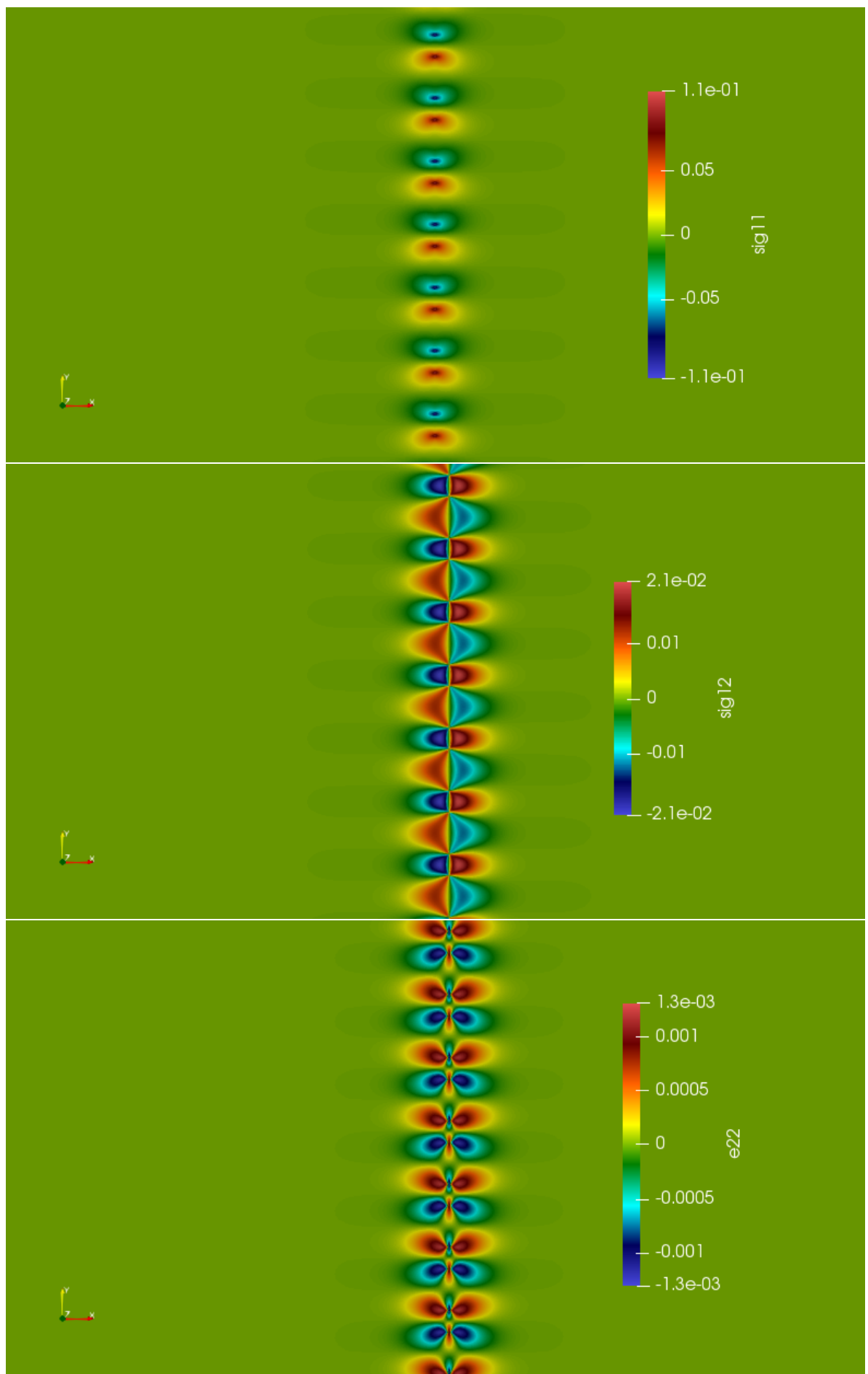

Fig. 5. Elastic fields for a STGB $\Sigma 41(540)[001]$ in Al considered as an array of disclination dipoles modeled by FFT-based method: normal stress $\sigma_{11}$ (top) and shear stress $\sigma_{12}$ (middle) normalized by $D \omega$, axial (elastic) strain $\varepsilon_{22}^{e}$ is also simulated (bottom). 


\subsection{Disconnection defect and phase boundary "terrace" in anisotropic bi-materials}

Disconnection defects and hetero-interfaces or martensitic interfaces can be modeled by combinations of dislocations and disclination dipoles. Hirth and Pond [1996, 2011] used a Burgers circuit to define the equivalent Burgers vector of the disconnection defect. This step character can be modeled using wedge disclination dipoles [Hirth et al., 2006]. Let us first consider a typical single disconnection defect embedded in an isotropic elastic elastic medium. The isotropic elastic constants of copper $(\mathrm{Cu})$ are used with Young's modulus $E=130 G P a$ and Poisson ratio $\nu=0.34$. The single disconnection defect is defined in three steps as a combination of rotation and displacement discontinuities (see Fig. 6 for details). Therefore, the resulting defect contains one wedge disclination dipole characterized by a non zero disclination density component with $\theta_{33}= \pm \omega / \delta^{2}$ and $\omega=\pi / 4 \mathrm{rad}$. The remaining edge dislocation is defined by a dislocation density tensor containing two non zero components: $\alpha_{31}=a \cos (\pi / 4) / \delta^{2}$ and $\alpha_{32}=a \sin (\pi / 4) / \delta^{2}$ where $a=0.3615 \mathrm{~nm}$ is the lattice parameter of $\mathrm{Cu}$. The computational grid is composed of $512 \times 512$ pixels with $\delta=0.11 \mathrm{~nm}$. Normal and shear stresses $\sigma_{11}$ and $\sigma_{12}$ normalized by the shear modulus $\mu$ are simulated by the FFT-based method and reported in Fig. 7. It is also found that the calculated elastic energy per unit volume for this disconnection defect is 1.7 times lower than an equivalent edge dislocation located at the center of the unit cell with Burgers vector $\mathbf{b}=\left(-3 \mathrm{ae}_{1}, 5 \mathrm{ae}_{\mathbf{2}}\right)$. Such result stems from more elastic screening for the disconnection in the case of disclination dipoles. 

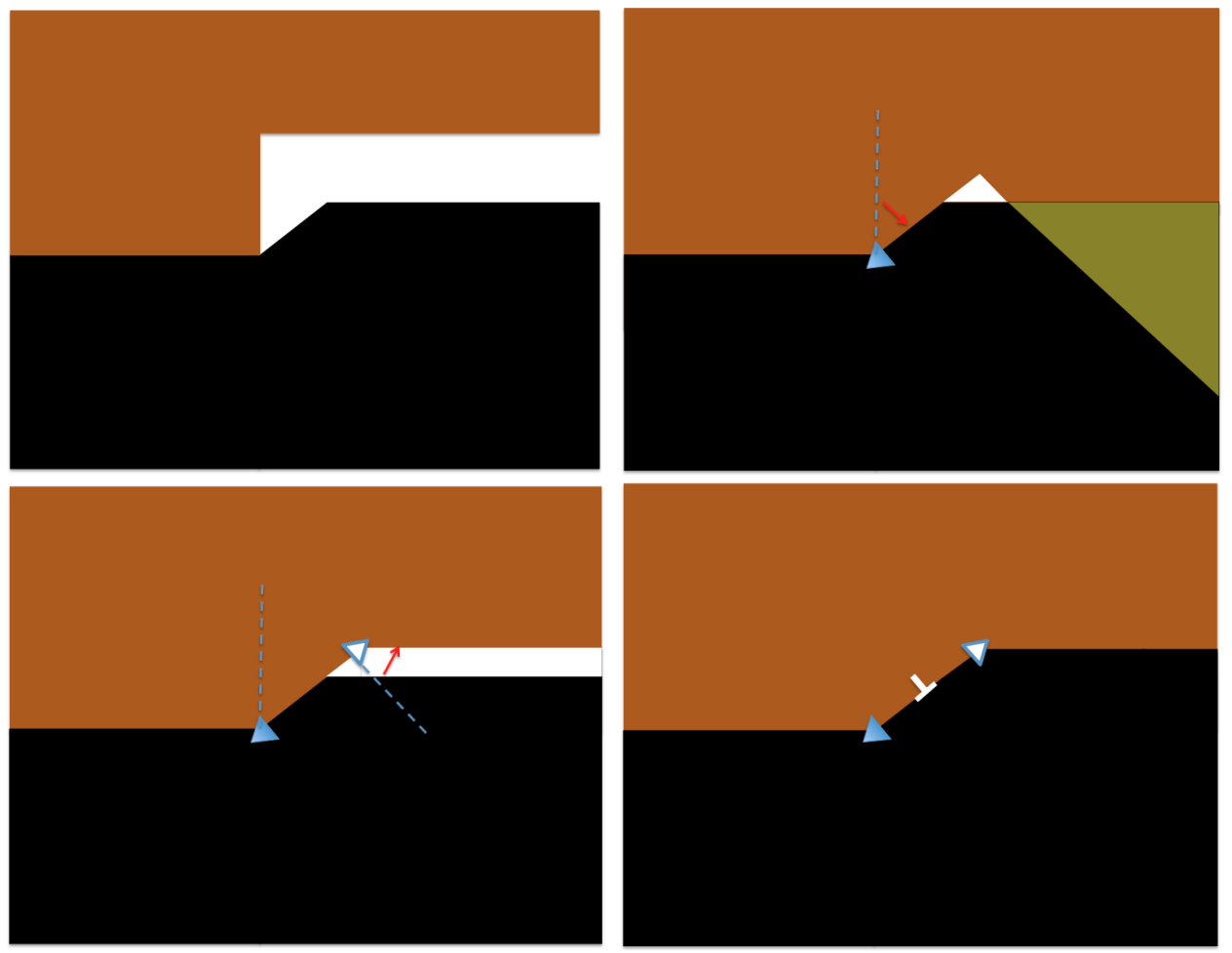

Fig. 6. Definition of a single disconnection defect: definition of material discontinuities between brown (upper) material and black (lower) material (top left), first step: a positive wedge disclination is introduced to define the rotational discontinuity on the lower part with a given angle (an overlap wedge occurs on the upper part with same angle) (top right), second step: a negative wedge disclination is introduced to eliminate the overlap wedge (bottom left), third step: an edge dislocation is introduced to define the displacement discontinuity (bottom right).

Some arrays of disconnections can also be used to model martensitic interfaces (habit planes) or hetero-interfaces forming a "terrace" structure observed with TEM (transmission electron microscopy) [Pond et al., 2003, 2007; Wang et al., 2011]. Following a case treated with atomistic simulations [Wang et al., 2011], a $\mathrm{Cu} / \mathrm{Ag}$ phase boundary "terrace" with anisotropic and heterogeneous elasticity is considered with the present numerical spectral approach. This configuration is represented in Fig. 8. The computational grid used for the FFT-based method is composed of $1024 \times 1024$ pixels with $\delta=0.11 \mathrm{~nm}$. The period of the defects along the "terrace" is given by $L=1.852 \mathrm{~nm}$, the angle between the "terrace" planes and the habit plane denoted $\theta$ (see Fig. 8) is given by $\theta=6.47^{\circ}$. Due to lattice mismatch between $\mathrm{Cu}$ and $\mathrm{Ag}\left(a_{C u}=0.3615 \mathrm{~nm}, a_{A g}=0.4085 \mathrm{~nm}\right)$ a misfit strain of $\varepsilon_{\text {mis } f i t}=0.122$ is present at the interface. Thus, the "terrace" first contains a periodic distribution of misfit dislocations (colored in orange) with a characteristic Burgers vector magnitude $b_{\text {misfit }}=0.236 \mathrm{~nm}$. In addition, the periodic step structure with con- 


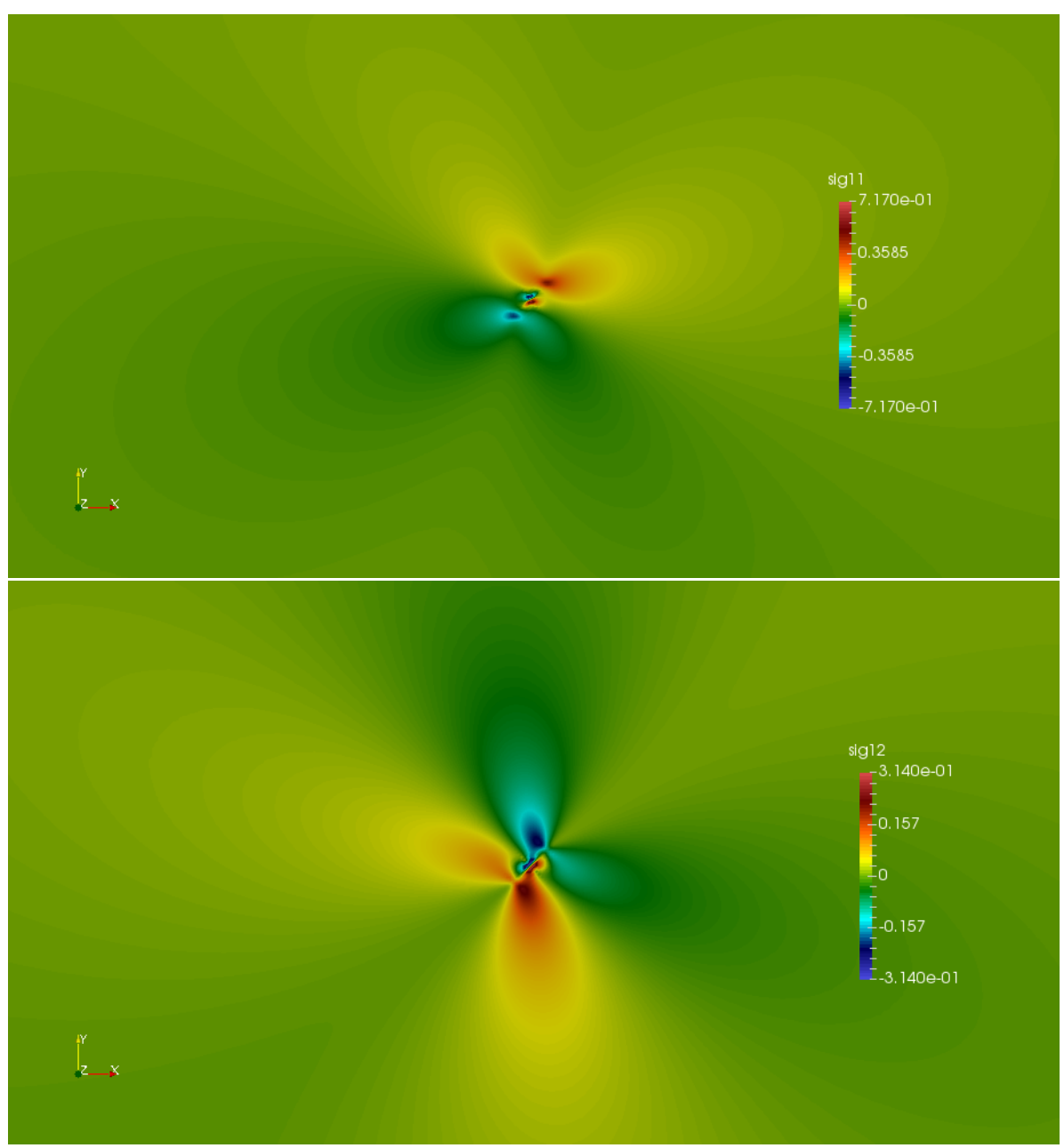

Fig. 7. Elastic fields for a single disconnection defect in $\mathrm{Cu}$ considered as a combination of a disclination dipole and a dislocation modeled by FFT-based method: normal stress (top) and shear stress (bottom) normalized by the shear modulus $\mu$.

stant step height $h=0.2084 \mathrm{~nm}$ is related to disconnection defects [Wang et al., 2011]. It is first represented by wedge disclination dipoles of arms fixed to $h / 2$ and with strength $\omega=5.33^{\circ}$ so that the disclination density components are given on one pixel by $\theta_{33}= \pm \omega / \delta^{2}$. Second, the disconnection is completed as previously by dislocations centered on the disclination dipoles with a Burgers vector given by bi-cristallography: $b_{d i s l o}=0.0274 \mathrm{~nm}$. The common crystallographic plane in the face centered cubic structure between both phases is the (111) plane (habit plane). For numerical calculations, anisotropic (cubic) elasticity in both $\mathrm{Cu}$ and $\mathrm{Ag}$ is considered. For $\mathrm{Cu}$, the elastic moduli defined in the cubic lattice frame are: $C_{11}=168.4 \mathrm{GPa}, C_{12}=121.4 \mathrm{GPa}, C_{44}=75.4 \mathrm{GPa}$ with an anisotropic factor of 
$A=2 C_{44} /\left(C_{11}-C_{12}\right)=3.21$. For Ag, the elastic moduli defined in the cubic lattice frame are: $C_{11}=124 \mathrm{GPa}, C_{12}=93.4 \mathrm{GPa}, C_{44}=46.1 \mathrm{GPa}$ with an anisotropic factor of $A=3.01$. The numerical convergence of the FFT-based algorithm is achieved when the error $\epsilon$ in Eq. 27 is smaller than $10^{-5}$. Because of low elastic moduli contrast between $\mathrm{Cu}$ and $\mathrm{Ag}$, the number of iterations for numerical calculation is only 6 . The numerical results on normal and shear stress fields are reported in Fig. 9 and for comparison the same fields are reported in Fig. 10 without the presence of misfit dislocations. It is found that the stress magnitudes are lower than the ones in Fig. 9. As a last remark, it is here highlighted that the numerical method is very fast for complex anisotropic and heterogeneous elasticity as the phase boundary "terrace" in anisotropic bi-materials. Even though the Fourier-based approach is developped in small deformation, the numerical results are consistent with the FEM results reported by Zhang et al. [2018] who used a more general framework in a finite deformation setting. Furthermore, the method is an alternative numerical method to analytical methods based on the sextic equation and Stroh formalism for anisotropic elasticity which was more developed for the dislocation theory [Stroh, 1958; Barnett and Lothe, 1974].

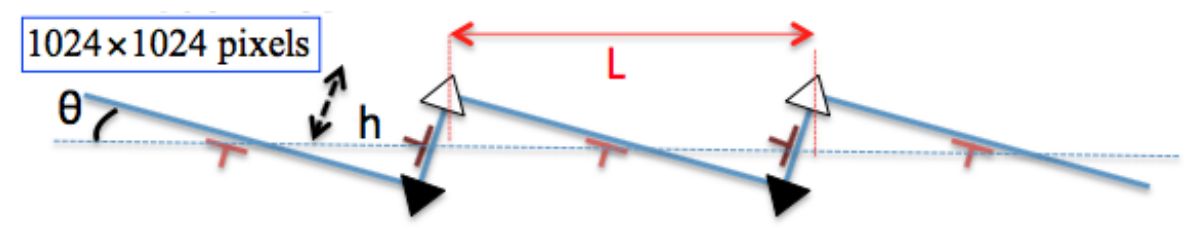

Fig. 8. $\mathrm{Cu} / \mathrm{Ag}$ phase boundary "terrace" configuration composed of an infinite periodic array of disconnections (each step in "terrace" is formed by a disclination dipole and a dislocation located in the center of the dipole) and misfit dislocations due to lattice mismatch at the interface of the anisotropic bi-material. 

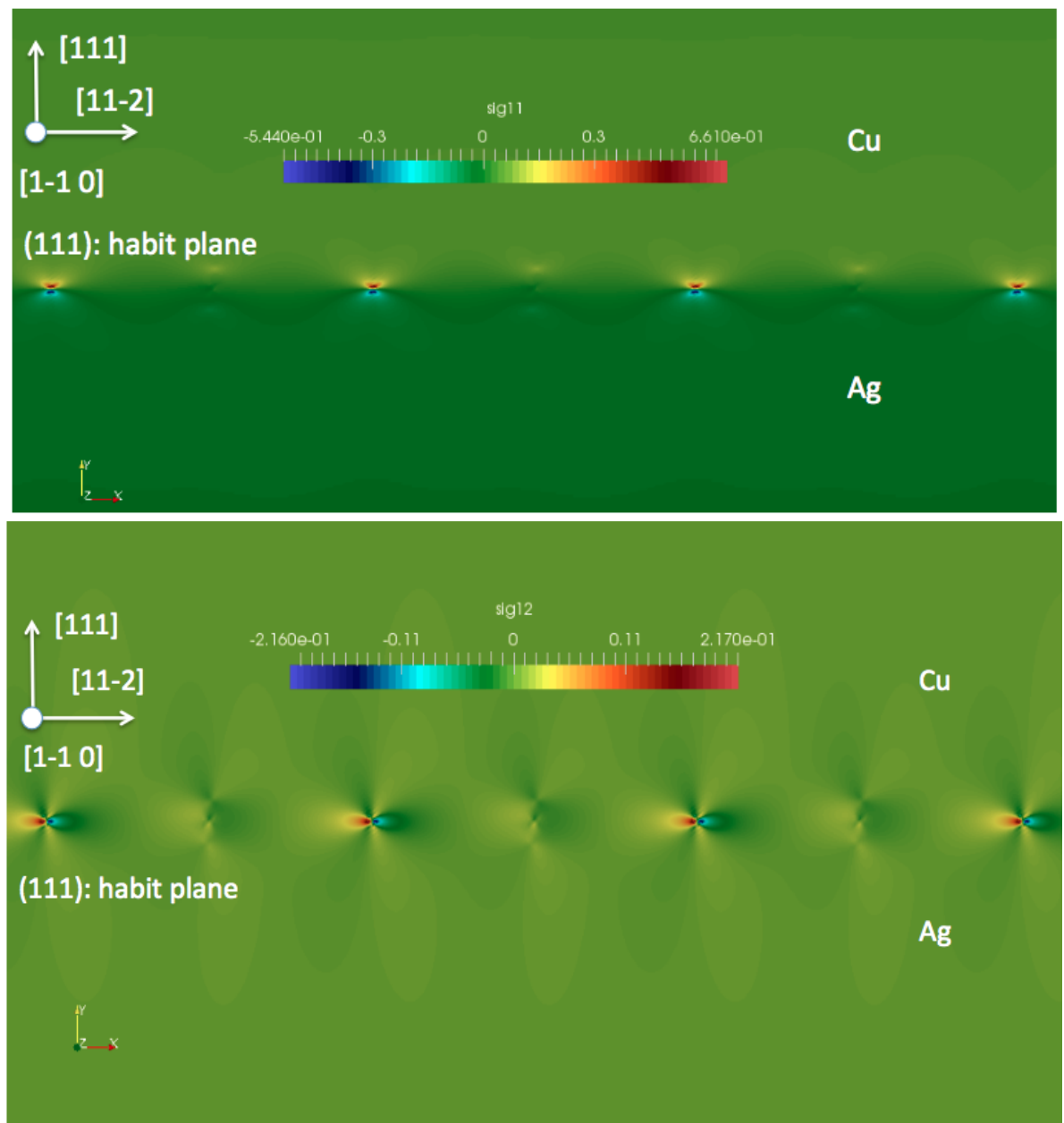

Fig. 9. Elastic fields for a $\mathrm{Cu} / \mathrm{Ag}$ phase boundary "terrace" with anisotropic and heterogeneous elasticity considered as a periodic array of disconnection defects in addition to misfit dislocations and simulated by the FFT-based method: normal stress (top) and shear stress (bottom) normalized by the shear modulus $\mu$.

\section{Concluding remarks and outlook}

This paper aimed at developing a numerical FFT-based general approach for continuum Field Dislocation and Disclination Mechanics (continuum defect mechanics) and for heterogeneous isotropic or anisotropic linear elastic media. After the identification of disclination and dislocation densities that are sources of incompatible elastic curvature and elastic strain, the numerical spectral approach was able to compute in a fast and efficient way (i.e. without numerical artefacts like spurious oscillations) the elastic fields of different bulk and interfacial defects. The numerically studied defects were disclination dipoles interacting with inhomogeneities, 

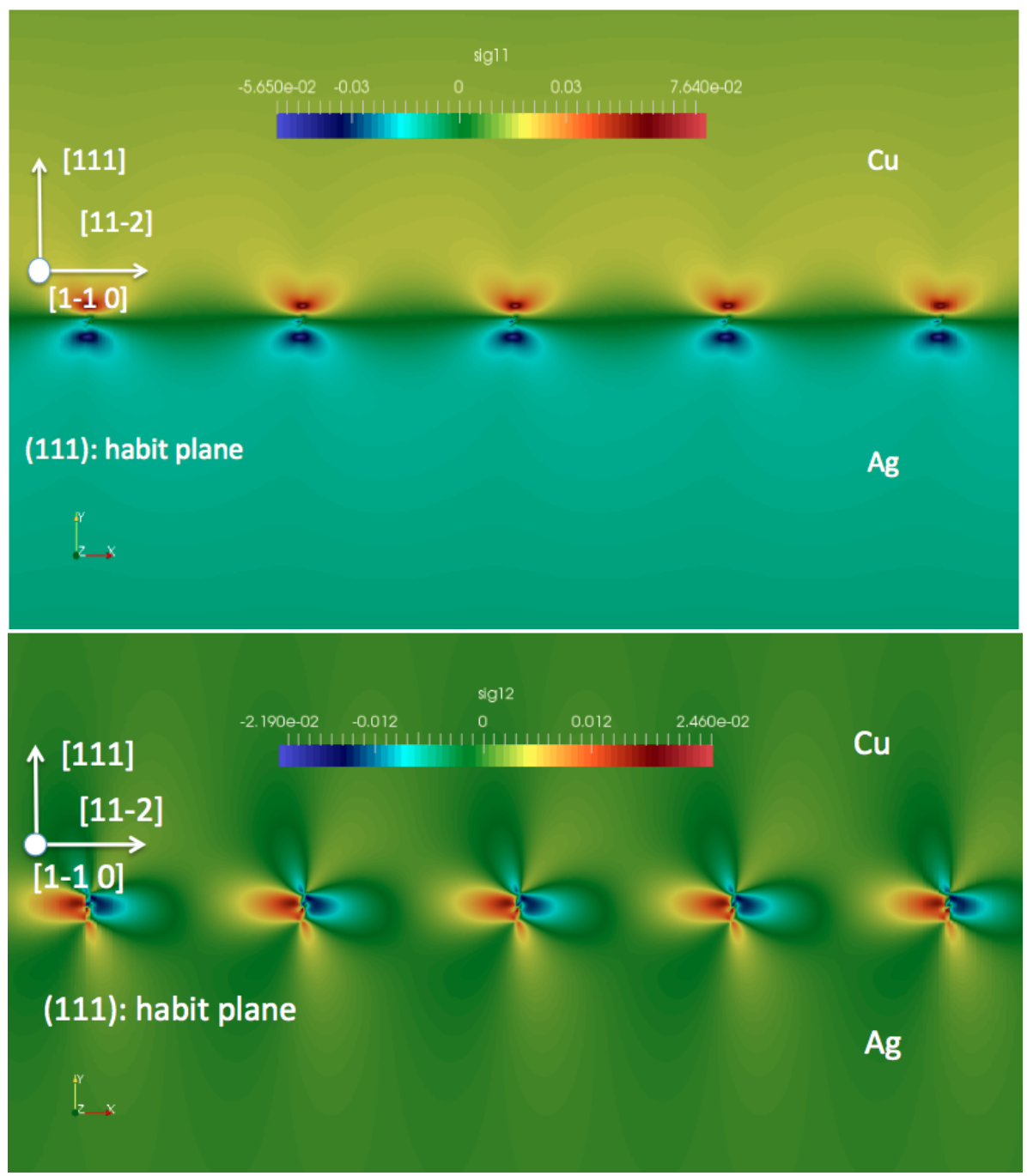

Fig. 10. Elastic fields for a $\mathrm{Cu} / \mathrm{Ag}$ phase boundary "terrace" with anisotropic and heterogeneous elasticity considered as a periodic array of disconnection defects without misfit dislocations and simulated by the FFT-based method: normal stress (top) and shear stress (bottom) normalized by the shear modulus $\mu$.

grain boundaries seen as DSUM, grain boundary disconnection defects and phase boundary "terraces" in anisotropic bi-materials. For heterogeneous cases, a few iterations were needed even for mechanical contrasts of 10 between the different phases were needed. Infinite contrasts like voids are also possible with the present $P_{C}-L S_{R}$ procedure but not rigid inhomogeneities [Willot, 2015; Djaka et al., 2017], which is left for further studies. The numerical FFT-based algorithm can also be accelerated with more recent refined gradient schemes [Schneider, 2017]. It is also of interest 
in forthcoming studies to extend the present FFT-based approach to more generalized micro-continuum field theories with disclination defects and with numerical calculations of configurational forces [Lazar and Maugin, 2007; Maugin, 2011] .

\section{Acknowledgements}

This work was supported by the French State through the program Investment in the future operated by the National Research Agency (ANR) and referenced by ANR-11-LABX-0008- 01 (Laboratory of Excellence 'DAMAS': Design of Alloy Metals for low-mAss Structures).

\section{References}

Acharya, A. [2001] "A model of crystal plasticity based on the theory of continuously distributed dislocations," J. Mech. Phys. Solids 49, 761-785.

Acharya, A. and Fressengeas, C. [2012] "Coupled phase transformations and plasticity as a field theory of deformation incompatibility," Int. J. Fract. 174, $87-94$.

Akarapu, S., Zbib, H. and Hirth, J. P. [2008] "Modeling and analysis of disconnections in tilt walls," Scripta Mater. 59, 265-267.

Anglin, B. S., Lebensohn, R. A. and Rollett, A. D. [2014] "Validation of a numerical method based on Fast Fourier Transforms for heterogeneous thermoelastic materials by comparison with analytical solutions," Comp. Mater. Sci. 87, 209-217.

Anthony, K. H. [1970] "Die Theorie der Disklinationen," Arch. Rat. Mech. Anal. 39, $43-88$.

Anthony, K. H., Essmann, U., Seeger, A. and Träuble, H. [1968] "Disclinations and the Cosserat Continuum with incompatible rotations," in Mechanics of Generalized Media. Proceedings of the IUTAM Symposium on the generalized Cosserat continuum and the continuum theory of dislocations with applications (Kröner, E. (ed.), Springer Verlag, Berlin), pp. 355-358.

Barnett, D. M. and Lothe, J. [1974] "An image force theorem for dislocations in anisotropic bicrystals," J. Phys. F 4, 1618-1635.

Beausir, B. and Fressengeas, F. [2013] "Disclination densities from EBSD orientation mapping," Int. J. Solids Struct. 503, 137-146.

Berbenni, S., Taupin, V., Djaka, K. S. and Fressengeas, C. [2014] "A numerical spectral approach for solving elasto-static Field Dislocation and G-Disclination Mechanics," Int. J. Solids Struct. 51, 4157-4175.

Berbenni, S., Taupin, V., Fressengeas, C. and Capolungo, L. [2016] "A fast Fourier transform-based approach for generalized disclination mechanics within a couple stress theory," Generalized Continua as Models for Classical and Advanced Materials, Advanced Structured Materials,H. Altenbach and S. Forest (eds.), Springer International Publishing Switzerland , 47-75. 
Bilby, B. A., Bullough, R. and Smith, E. [1955] "Continuous distributions of dislocations: a new application of the methods of non-riemannian geometry," Proc. Roy. Soc. London A 231, 263-273.

Bollmann, W. [1991] "The stress field of a model triple-line disclination," Mater. Sci. Eng. A 136, 1-7.

Brenner, R., Beaudoin, A. J., Suquet, P. and Acharya, A. [2014] "Numerical implementation of static Field Dislocation Mechanics theory for periodic media," Philos. Mag. 94, 1764-1787.

Brisard, S. and Dormieux, L. [2010] "FFT-based methods for the mechanics of composites: A general variational framework," Comp. Mater. Sci. 49, 663671.

deWit, R. [1970] "Linear theory of static disclinations," in Fundamental aspects of dislocation theory (Simmons, J.A., de Wit, R., Bullough, R. NBS Spec. Publ. 317, vol. 1, (National Bureau of Standards, Washington, DC)), pp. 651-680.

deWit, R. [1973a] "Theory of disclinations: II. Continuous and Discrete Disclinations in Anisotropic Elasticity," J. Res. Nat. Bureau of Standards, A. Physics and Chemistry 77A(1), 49-100.

deWit, R. [1973b] "Theory of disclinations: IV. Straight disclinations," J. Res. Nat. Bureau of Standards, A. Physics and Chemistry 77A(5), 607-658.

Dingreville, R. and Berbenni, S. [2016] "On the interaction of solutes with grain boundaries," Acta Mater. 104, 237-249.

Djaka, K. S., Taupin, V., Berbenni, S. and Fressengeas, C. [2015] "A numerical spectral approach to solve the dislocation density transport equation," Modell. Simul. Mater. Sci. Eng. 23, 065008 (27pp).

Djaka, K. S., Villani, A., Taupin, V., Capolungo, L. and Berbenni, S. [2017] "Field dislocation mechanics for heterogeneous elastic materials: A numerical spectral approach," Comput. Methods Appl. Mech. Engrg. 315, 921-942.

Dreyer, W., Müller, W. H. and Olschewski, J. [1999] "An approximate analytical $2 \mathrm{D}$-solution for the stresses and strains in eigenstrained cubic materials," Acta Mech. 136(3-4), 171-192.

Dundurs, J. and Sendeckyj, G. P. [1965] "Edge dislocation inside a circular inclusion," J. Mech. Phys. Solids 13, 141-147.

Eshelby, J. D. [1957] "The determination of the elastic field of an ellipsoidal inclusion and related problems," Proc. Roy. Soc. London A 241, 376-396.

Eshelby, J. D. [1961] "Elastic inclusions and inhomogeneities," in Progress in Solids Mechanics (Vol. II) (Sneddon, I.N., Hill, R. (eds.), North Holland, Amsterdam), pp. 88-140.

Fressengeas, C., Taupin, V. and Capolungo, L. [2011] "An elasto-plastic theory of dislocation and disclination fields," Int. J. Solids Struct. 48, 3499-3509.

Fressengeas, C., Taupin, V. and Capolungo, L. [2014] "Continuous modeling of the structure of symmetric tilt boundaries," Int. J. Solids Structures 51, 14341441. 
Frigo, M. and Johnson, S. G. [1998] "An adaptive software architecture for the FFT," Proc. Int. Conf. Acoust. Speech Sig. Process. 3, 1381-1384.

Gertsman, V. Y., Nazarov, A. A., Romanov, A. E., Valiev, R. Z. and Vladimirov, V. I. [1989] "Disclination-structural unit model of grain boundaries," Philos. Mag. A 59(5), 1113-1118.

Gutkin, M. Y. and Ovid'ko, I. A. [2003] "Disclinations and rotational deformation in nanocrystalline materials," Rev. Adv. Mater. Sci. 4, 79-113.

Gutkin, M. Y. and Ovid'ko, I. A. [2004] Plastic Deformation in Nanocrystalline Materials (Springer, Berlin).

Hirth, J. P. and Lothe, J. [1982] Theory of Dislocations (2nd ed.) (Wiley, New York).

Hirth, J. P. and Pond, R. C. [1996] "Steps, dislocations and disconnections as interface defects relating to structures and phase transformations," Acta Mater. 44, 4749-4763.

Hirth, J. P. and Pond, R. C. [2011] "Compatibility and accommodation in displacive phase transformations," Prog. Mater. Sci. 56, 586-636.

Hirth, J. P., Pond, R. C. and Lothe, J. [2006] "Disconnections in tilt walls," Acta Mater. 54, 4237-4245.

Huang, W. and Mura, T. [1969] "Elastic fields and energies of a circular edge disclination and a straight screw disclination," J. Appl. Phys. 41, 5175-5179.

Hurtado, J. A., Elliot, B. R., Shodja, H. M., Gorelikov, D. V., Campbell, C. E., Lippard, H. E., Isabell, T. C. and Weertman, J. [1995] "Disclination grain boundary model with plastic deformation by dislocations," Mater. Sci. Eng. A 190, 1-7.

Khater, H. A., Serra, A., Pond, R. C. and Hirth, J. P. [2012] "The disconnection mechanism of coupled migration and shear at grain boundaries," Acta Mater. 60, 2007-2020.

Kossecka, E. and DeWit, R. [1977] "Disclination dynamics," Arch. Mech. 29, 749767.

Kröner, E. [1955] "Der fundamentale Zusammenhang zwischen Versetzungsdichte und Spannungsfunktionen," Z. Phys. 142, 463-475.

Kröner, E. [1958] Kontinuumstheorie der Versetzungen und Eigenspannungen (Collatz L and Loesch F (eds.). Ergebnisse der Angewewandte Mathematik 5, Springer Verlag, Berlin).

Kröner, E. [1981] "Continuum theory of defects," in Physics of defects (R. Balian and al. (Eds.), Les Houches, Session 35, North Holland, New York), pp. 215315 .

Kröner, E. [1990] Modified Green functions in the theory of heterogeneous and/or anisotropic linearly elastic media (ed. G.J. Weng, M. Taya, M. Abe, Springer Verlag, Berlin Germany).

Lardner, R. H. [1969] "Dislocation dynamics and the theory of the plasticity of single crystals," ZAMP 20, 514-529.

Lazar, M. [2016] "Micromechanics and dislocation theory in anisotropic elasticity," 
Journal of Micromechanics and Molecular Physics 1, 1650011-(22p).

Lazar, M. [2017] "Micromechanics and theory of point defects in anisotropic elasticity: Eshelby factor meets Eshelby tensor," Journal of Micromechanics and Molecular Physics 2, 1750005-(19p).

Lazar, M. and Maugin, G. A. [2007] "On microcontinuum field theories: the Eshelby stress tensor and incompatibility conditions," Philos. Mag. 87, 3853-3870.

Lebensohn, R. A. [2001] "N-site modeling of a 3D viscoplastic polycrystal using Fast Fourier Transform," Acta Mater. 49, 2723-2737.

Lebensohn, R. A. and Needleman, A. [2016] "Numerical implementation of non-local polycrystal plasticity using fast Fourier transforms," J. Mech. Phys. Solids $\mathbf{9 7}$, 333-351.

Leff, A. C., Weinberger, C. R. and Taheri, M. L. [2017] "On the accessibility of the disclination tensor from spatially mapped orientation data," Acta Mater 138, 161-173.

Li, J. C. M. [1972] "Disclination model of high angle grain boundaries," Surf. Sci. 31, 12-26.

Liu, Y. M., Fang, Q. H. and Jiang, C. P. [2006] "A wedge disclination dipole interacting with a circular inclusion," Phys. Status Solidi (a) 203, 443-458.

Maugin, G. A. [2011] Configurational forces: thermomechanics, physics, mathematics, and numerics (CRC Press).

Moulinec, H. and Suquet, P. [1994] "A fast numerical method for computing the linear and non linear properties of composites," C. R. Acad. Sci. Paris II 318, $1417-1423$.

Moulinec, H. and Suquet, P. [1998] "A numerical method for computing the overall response of nonlinear composites with complex microstructure," Comput. Methods Appl. Mech. Engrg. 157, 69-94.

Müller, W. H. [1996] "Mathematical vs. experimental stress analysis of inhomogeneities in solids," J. Phys. IV 6(C1), 139-148.

Müllner, P. and King, A. H. [2016] "Deformation of hierarchically twinned martensite," Acta Mater. 58, 5242-5261.

Mura, T. [1963] "Continuous distribution of moving dislocations," Philos. Mag. 89, 843-857.

Mura, T. [1972] "Semi-microscopic plastic distortion and disclinations," Arch. Mech. Stos. 24, 449-456.

Mura, T. [1987] Micromechanics of defects in solids (Kluwer Academic Publishers, Dordrecht, The Netherlands).

Nazarov, A. A. and Romanov, A. E. [1989] "On the average misorientation angle of general tilt boundaries," Philos. Mag. Lett. 60(5), 187-193.

Nazarov, A. A., Shenderova, O. A. and Brenner, D. W. [2000] "On the disclinationstructural unit model of grain boundaries," Mater. Sci. Eng. A 281(1), 148155 .

Nye, J. F. [1953] "Some geometrical relations in dislocated crystals," Acta Metall. 
1, 153-162.

Pond, R. C., Celotto, S. and Hirth, J. P. [2003] "A comparison of the phenomenological theory of martensitic transformations with a model based on interfacial defects," Acta Mater. 51, 5385-5398.

Pond, R. C., Ma, X., Hirth, J. P. and Mitchell, T. E. [2007] "Disconnections in simple and complex structures," Philos. Mag. 87, 5289-5307.

Rajabzadeh, A. [2013] Experimental and theoretical study of shear-coupled grain boundary migration ( $\mathrm{PhD}$ Thesis, University of Toulouse, France).

Rajabzadeh, A., Mompiou, F., Lartigue-Korinek, S., Combe, N. and Legros, M. [2014] "The role of disconnections in deformation-coupled grain boundary migration," Acta Mater. 77, 223-235.

Ren, Y. and Cao, G. [2016] "Effect of geometrical defects on the tensile properties of graphene," Carbon 103, 125-133.

Romanov, A. E. and Kolesnikova, A. L. [2009] "Application of disclination concept to solid structures," Prog. Mater. Sci. 54(6), 740-769.

Romanov, A. E. and Vladimirov, V. [1992] "Disclinations in crystalline solids (chap. 47)," in Dislocations in Solids (vol. 9) (Elsevier Science Publishers B.V.), pp. 191-402.

Roy, A. and Acharya, A. [2005] "Finite element approximation of field dislocation mechanics," J. Mech. Phys. Solids 53, 143-170.

Schaefer, H. [1968] "The basic affine connection in a Cosserat Continuum," in $\mathrm{Me}$ chanics of Generalized Media. Proceedings of the IUTAM Symposium on the generalized Cosserat continuum and the continuum theory of dislocations with applications (Kröner, E. (ed.), Springer Verlag, Berlin), pp. 57-62.

Schneider, M. [2017] "An FFT-based fast gradient method for elastic and inelastic unit cell homogenization problems," Comp. Meth. Appl. Mech. Eng. 315, 846866.

Seefeldt, M., Delannay, L., Peeters, B., Aernoudt, E. and Van Houtte, P. [2001] "Modeling the initial stage of grain subdivision with the help of a coupled substructure and texture evolution algorithm," Acta Mater 49, 2129-2143.

Simmons, J. and Bullough, R. [1970] "Internal stress and the incompatibility problem in infinite anisotropic elasticity," in Fundamental aspects of dislocation theory (Simmons, J.A., de Wit, R., Bullough, R. NBS Spec. Publ. 317, vol. 1, (National Bureau of Standards, Washington, DC)), pp. 89-124.

Stroh, A. N. [1958] "Dislocations and cracks in anisotropic elasticity," Philos. Mag. 3, 625-646.

Sutton, A. P. and Vitek, V. [1983] "On the structure of tilt grain boundaries in cubic metals I. Symmetrical tilt boundaries," Philosophical Transactions of the Royal Society of London A: Mathematical, Physical and Engineering Sciences 309(1506), 1-36.

Taupin, V., Capolungo, L., Fressengeas, C., Das, A. and Upadhyay, M. [2013] "Grain boundary modeling using an elasto-plastic theory of dislocation and disclina- 
tion fields," J. Mech. Phys. Solids 61, 370-384.

Taupin, V., Gbemou, K., Fressengeas, C. and Capolungo, L. [2017] "Nonlocal elasticity tensors in dislocation and disclination cores," J. Mech. Phys. Solids 100, 62-84.

Teodosiu, C. [1982] Elastic models of crystal defects (Springer, Berlin).

Upadhyay, M., Capolungo, L., Taupin, V. and Fressengeas, C. [2013] "Elastic constitutive laws for incompatible crystalline media: the contributions of dislocations, disclinations and g-disclinations," Philos. Mag. 93, 794-832.

Upadhyay, M. V., Capolungo, L., Taupin, V. and Fressengeas, C. [2011] "Grain boundary and triple junction energies in crystalline media: a disclination based approach," Int. J. Solids Struct. 48(22), 3176-3193.

Vinogradov, V. and Milton, G. W. [2008] "An accelerated FFT algorithm for thermoelastic and non-linear composites," Int. J. Num. Meth. Eng. 76, 1678-1695.

Volterra, S. [1907] "Sur l'équilibre des corps élastiques multiplement connexes," Ann. Sci. Ecol. Norm. Sup. III 24, 401-517.

Wang, J., Hirth, J. P., Pond, R. C. and Howe, J. M. [2011] "Rotational partioning at two-phase interfaces," Acta Mater. 59, 241-251.

Willis, J. R. [1967] "Second-order effects of dislocations in anisotropic crystals," Int. J. Eng. Sci. 5, 171-190.

Willis, J. R. [1981] "Variational and related methods for the overall properties of composites," Adv. Appl. Mech. 21, 1-78.

Willot, F. [2015] "Fourier-based schemes for computing the mechanical response of composites with accurate local fields," C. R. Mecanique 343, 232-245.

Willot, F. and Pellegrini, Y. P. [2008] "Fast Fourier transform computations and build-up of plastic deformation in 2D, elastic-perfectly plastic, pixelwise disordered porous media," Continuum Models and Discrete Systems, D. Jeulin and S. Forest (eds.), CMDS11, Ecole des Mines Paris , 443-449.

Wu, J. and Wei, Y. [2013] "Grain misorientation and grain-boundary rotation dependent mechanical properties in polycrystalline graphene," J. Mech. Phys. Solids 61, 1421-1432.

Zhang, C., Acharya, A. and Puri, S. [2018] "Finite element approximation of the fields of bulk and interfacial line defects," J. Mech. Phys. Solids 114, 258-302.

Zubov, L. M. [1997] Nonlinear theory of dislocations and disclinations on elastic bodies (Springer, Berlin). 